\title{
On-site correlation in valence and core states of ferromagnetic nickel
}

\author{
F. Manghi, V. Bellini \\ Istituto Nazionale per la Fisica della Materia and \\ Dipartimento di Fisica, Università di Modena, Via Campi 213/a, I-41100 Modena, Italy \\ C. Arcangeli \\ Max-Planck-Institut für Festkörperforschung, Heisenbergstr. 1, D-70569 Stuttgart, Germany
}

\begin{abstract}
We present a method which allows to include narrow-band correlation effects into the description of both valence and core states and we apply it to the prototypical case of nickel. The results of an ab-initio band calculation are used as input mean-field eigenstates for the calculation of self-energy corrections and spectral functions according to a three-body scattering solution of a multi-orbital Hubbard hamiltonian. The calculated quasi-particle spectra show a remarkable agreement with photoemission data in terms of band width, exchange splitting, satellite energy position of valence states, spin polarization of both the main line and the satellite of the $3 p$ core level.

PACS numbers: 71.10.-w, 79.60.-i
\end{abstract}

\section{INTRODUCTION}

It is well established that the description of electronic states in narrow band materials requires improvements over the single particle approximation with a proper inclusion of on-site Coulomb interaction between localized electrons [1]. In these systems the itinerant character of valence electrons which is clearly shown by the energy k-dispersion observed in photoemission spectroscopy coexists with strong local electronic correlation responsible of other observed features such as satellite structures and band-narrowing effects. The interplay of localization and itineracy has also been indicated as a possible explanation of the observed spin polarization of core level spectra through exchange coupling betweeen localized (core) and itinerant (valence) states [2,3].

In this paper we present a theoretical description of the valence and core electron states of nickel according to a method recently developed which has been designed to treat highly correlated and highly hybridized systems [4] including both the itinerant character of band electrons and the strong localized electron-electron repulsion. This method allows to include narrow-band correlation effects into a first-principle band calculation; the single-particle band states are determined according to the density functional theory in the local density approximation (LDA) and the correlation effects are described as a 3-body scattering (3BS) solution of a multi-orbital Hubbard hamiltonian. This approach has been previously applied to the description of valence and conduction states of both model systems [5,6] and realistic materials 4,6 , 8 . We present here an extension of the method in order to treat both valence and core state on the same footing; this extension is possible in this scheme since it relies on a multi-orbital Hubbard hamiltonian where core and valence states can coexist and on the 3BS method which can be applied for any value of effective on-site electron-electron repulsion.

As far as the valence states are concerned various methods have been proposed to augment conventional band theory for the description of the electronic states of nickel; some of them are based on perturbative expansion either in the e-e interaction (GW approach [9], second- order solution of the Hubbard hamiltonian [10, 11, 12]) or in the fluctuations of the electron occupation around LDA mean-field solution [13]; others apply a t-matrix scheme where the effect of electron correlation on one electron removal energies from a partially filled band is described as a hole-hole interaction [14.15. This method is strictly valid only in the limit of an almost filled band (dilute limit) and its application to the case of nickel has been questioned [10]. In order to implement this approach it is necessary to include also electronelectron scattering channels and to solve a 3-body scattering problem involving two holes and one electron. This is the spirit of the 3BS theory we apply here and which originally has been formulated by Igarashi [5]. This approach has recently been applied to the description of valence states of nickel [16] choosing, however, an approximate form of self-energy in terms of the antisymmetrized vertex function; in this way the self-energy turns out to be real, giving rise to peaks in the spectral density of unphysical zero width. Here we will instead adopt a version of the theory which avoids this shortcoming and which is based on the explicit solution of the 3-body scattering equations [4, 6, ], 7].

The interpretation of core level spectroscopies has up to now largely been based on atomic models which interpret the structures observed in the one electron removal spectra in terms of multiplet states formed by coupling the core hole to the unfilled valence shell [17]. This scheme has in particular been applied to the photoemission spectra from the core states of transition metals [18] attributing the observed characteristic line splitting to intra-atomic exchange 
interaction between core and valence electrons of an isolated atom. Such an approach has been seriously questioned since the observed splittings and the energy scale of the interaction are of the same order of magnitude as the valence band width [2]; a picture which takes into account the itinerant character of valence electrons seems therefore necessary. With the advent of spin polarized spectroscopies such as spin resolved x-ray photoemission [3,19] and magnetic circular dichroism 20 the spin dependence of both the main line and the satellites of $\mathrm{Ni}$ core level spectra has been widely investigated [19,21,22, 23]. We will show that the 3BS solution of a multi-band Hubbard hamiltonian, where full details of the valence band structure are included, can account for these spectroscopical features and interpret them in terms of on-site interaction between localized (core) and itinerant (valence) states.

The paper is organized as follows: we present in section II the multi-orbital Hubbard hamiltonian and its relationship to the band hamiltonian we want to implement; section IIII describes the main characteristics of the method which we use to get an approximate solution of the Hubbard hamiltonian in terms of self-energy corrections to band eigenstates and of spectral densities; section [IV and $\mathrm{V}$ specialize to the case of valence and core states respectively; the results and the comparison with experiments are presented in section $\mathrm{VI}$.

\section{MULTI-BAND HUBBARD HAMILTONIAN}

Band structure eigenvalues are in many cases good zero-order approximations to the excitation spectrum of a solid and it seems reasonable to use them as a starting point for the inclusion of correlation effects according to the Hubbard model; the implicit assumption is that among all the many body terms responsible of electron correlation, the Coulomb repulsion between electrons on the same site is the one which needs to be treated explicitly. To do this it is necessary to define precisely the relationship between band and Hubbard hamiltonians. Let us consider first a localized basis set $\phi_{i \alpha \sigma}(\mathbf{r}, s)$ with $i$ labelling the localization site, $\alpha$ the orbital character, $s$ and $\sigma$ the spin coordinate and eigenvalue respectively. The full many-body hamiltonian in second quantization is

$$
\hat{H}=\sum_{i \alpha \sigma} \epsilon_{i \alpha \sigma}^{0} \hat{n}_{i \alpha \sigma}+\sum_{\alpha \beta \sigma} \sum_{i j} t_{i \alpha, j \beta} \hat{c}_{i \alpha \sigma}^{\dagger} \hat{c}_{j \beta \sigma}+\frac{1}{2} \sum_{i \alpha j \beta l \gamma m \delta} \sum_{\sigma \sigma^{\prime}} V_{i \alpha \sigma, j \beta \sigma^{\prime}, l \gamma \sigma^{\prime}, m \delta \sigma} \hat{c}_{i \alpha \sigma}^{\dagger} \hat{c}_{j \beta \sigma^{\prime}}^{\dagger} \hat{c}_{l \gamma \sigma^{\prime}} \hat{c}_{m \delta \sigma}
$$

with $\hat{n}_{i \alpha \sigma}=\hat{c}_{i \alpha \sigma}^{\dagger} \hat{c}_{i \alpha \sigma}$ and $\hat{c}_{i \alpha \sigma}, \hat{c}_{i \alpha \sigma}^{\dagger}$ destruction and creation operators.

Here $\epsilon_{i \alpha \sigma}^{0}$ and $t_{i \alpha, j \beta}$ are the intra- and inter-atomic matrix elements of the one-particle hamiltonian ( kinetic energy + ionic potential), while $V_{i \alpha \sigma, j \beta \sigma^{\prime}, l \gamma \sigma^{\prime}, m \delta \sigma}$ are multi-center integrals involving the electron-electron interaction

$$
V_{i \alpha \sigma, j \beta \sigma^{\prime}, l \gamma \sigma^{\prime}, m \delta \sigma}=\sum_{s s^{\prime}} \int \phi_{i \alpha \sigma}^{*}(\mathbf{r}, s) \phi_{j \beta \sigma^{\prime}}^{*}\left(\mathbf{r}^{\prime}, s^{\prime}\right) \frac{e^{2}}{\left|\mathbf{r}-\mathbf{r}^{\prime}\right|} \phi_{l \gamma \sigma^{\prime}}\left(\mathbf{r}^{\prime}, s^{\prime}\right) \phi_{m \delta \sigma}(\mathbf{r}, s) d \mathbf{r} d \mathbf{r}^{\prime}
$$

In this last expression the dominant contribution comes from the one-center integrals with $i=j=l=m$ which are the usual on-site Coulomb term

$$
U_{\alpha \beta}^{i}=V_{i \alpha \sigma, i \beta \sigma, i \beta \sigma, i \alpha \sigma}=V_{i \alpha \sigma, i \beta-\sigma, i \beta-\sigma, i \alpha \sigma}
$$

and exchange term

$$
J_{\alpha \beta}^{i}=V_{i \alpha \sigma, i \beta \sigma, i \alpha \sigma, i \beta \sigma}
$$

The full many body hamiltonian can then be written as

$$
\begin{aligned}
\hat{H} & =\sum_{i \alpha \sigma} \epsilon_{i \alpha \sigma} \hat{n}_{i \alpha \sigma}+\sum_{\alpha \beta \sigma} \sum_{i j} t_{i \alpha, j \beta} \hat{c}_{i \alpha \sigma}^{\dagger} \hat{c}_{j \beta \sigma} \\
& +\frac{1}{2} \sum_{\alpha \beta}\left[\sum_{i}\left(U_{\alpha \beta}^{i}-J_{\alpha \beta}^{i}\right) \sum_{\sigma} \hat{n}_{i \alpha \sigma} \hat{n}_{i \beta \sigma}+\sum_{i} U_{\alpha \beta}^{i} \sum_{\sigma} \hat{n}_{i \alpha \sigma} \hat{n}_{i \beta-\sigma}\right] \\
& +\ldots \text { (multi-center terms) }
\end{aligned}
$$

Different approximations of the exact hamiltonian (2.1) can be obtained using a mean field approach which amounts to neglect fluctuations in the electron occupation

$$
\begin{aligned}
\hat{n}_{i \alpha \sigma} \hat{n}_{i \beta \sigma^{\prime}} & =\hat{n}_{i \alpha \sigma}<\hat{n}_{i \beta \sigma^{\prime}}>+\hat{n}_{i \beta \sigma^{\prime}}<\hat{n}_{i \alpha \sigma}>-<\hat{n}_{i \alpha \sigma}><\hat{n}_{i \beta \sigma^{\prime}}> \\
& +\left(\hat{n}_{i \alpha \sigma^{\prime}}-<\hat{n}_{i \alpha \sigma}>\right) \cdot\left(\hat{n}_{i \beta \sigma^{\prime}}-<\hat{n}_{i \beta \sigma^{\prime}}>\right) \\
& \simeq \hat{n}_{i \alpha \sigma}<\hat{n}_{i \beta \sigma^{\prime}}>+\hat{n}_{i \beta \sigma^{\prime}}<\hat{n}_{i \alpha \sigma}>-<\hat{n}_{i \alpha \sigma}><\hat{n}_{i \beta \sigma^{\prime}}>
\end{aligned}
$$


where $\langle. .>$ means a ground state average. The mean-field approximation can be applied to all the many body terms of (2.1) transforming it into a single-particle hamiltonian

$$
\hat{H}^{M F}=\sum_{i \alpha \sigma} \epsilon_{i \alpha \sigma}^{M F} \hat{n}_{i \alpha \sigma}+\sum_{\alpha \beta \sigma} \sum_{i j} t_{i \alpha, j \beta} \hat{c}_{i \alpha \sigma}^{\dagger} \hat{c}_{j \beta \sigma}
$$

Any band structure calculation, where the interacting system is described as an effective single-particle problem, corresponds to the self-consistent solution of $\hat{H}^{M F}$. Another possibility is to apply the mean field approximation selectively to the multi-center integrals, keeping the full many body character in the one-center terms; in this way one gets a generalized Hubbard model

$$
\begin{aligned}
\hat{H}^{H} & =\sum_{i \alpha \sigma} \epsilon_{i \alpha \sigma}^{H} \hat{n}_{i \alpha \sigma}+\sum_{\alpha \beta \sigma} \sum_{i j} t_{i \alpha, j \beta} \hat{c}_{i \alpha \sigma}^{\dagger} \hat{c}_{j \beta \sigma} \\
& +\frac{1}{2} \sum_{\alpha \beta}\left[\sum_{i}\left(U_{\alpha \beta}^{i}-J_{\alpha \beta}^{i}\right) \sum_{\sigma} \hat{n}_{i \alpha \sigma} \hat{n}_{i \beta \sigma}+\sum_{i} U_{\alpha \beta}^{i} \sum_{\sigma} \hat{n}_{i \alpha \sigma} \hat{n}_{i \beta-\sigma}\right]
\end{aligned}
$$

Since $H^{M F}$ and $H^{H}$ differ for the treatment of the on-site correlation - included in $H^{M F}$ as a mean-field and treated as a many body term in $H^{H}$ - it is easy to show that

$$
\epsilon_{i \alpha \sigma}^{M F}=\epsilon_{i \alpha \sigma}^{H}+\sum_{\beta}\left[\left(U_{\alpha \beta}^{i}-J_{\alpha \beta}^{i}\right)<\hat{n}_{i \beta \sigma}>+U_{\alpha \beta}^{i}<\hat{n}_{i \beta-\sigma}>\right]
$$

Due to the translational periodicity we can introduce an extended Bloch basis set

$$
\psi_{\mathbf{k} \sigma}^{n}(\mathbf{r}, s)=\frac{1}{\sqrt{N}} \sum_{i \alpha} C_{\alpha \sigma}^{n}(\mathbf{k}) e^{i \mathbf{k} \cdot \mathbf{R}_{i}} \phi_{i \alpha \sigma}(\mathbf{r}, s)
$$

and the corresponding relations for creation/destruction operators of electrons with wave vector $\mathbf{k}$, spin $\sigma$ and band index $n$

$$
\begin{gathered}
\hat{a}_{\mathbf{k} \sigma}^{n}=\frac{1}{\sqrt{N}} \sum_{i \alpha} C_{\alpha \sigma}^{n}(\mathbf{k}) e^{i \mathbf{k} \cdot \mathbf{R}_{i}} \hat{c}_{i \alpha \sigma} \\
\hat{a}_{\mathbf{k} \sigma}^{n^{\dagger}}=\frac{1}{\sqrt{N}} \sum_{i \alpha} C_{\alpha \sigma}^{n}(\mathbf{k})^{*} e^{-i \mathbf{k} \cdot \mathbf{R}_{i}} \hat{c}_{i \alpha \sigma}^{\dagger}
\end{gathered}
$$

Here $C_{\alpha}^{n}(\mathbf{k} \sigma)$ are the expansion coefficients of Bloch states in terms of localized orbitals, $N$ the number of unit cells. $H^{H}$ becomes

$$
\begin{aligned}
\hat{H}^{H}= & \sum_{\mathbf{k} n \sigma} \epsilon_{\mathbf{k} n \sigma}^{H} \hat{a}_{\mathbf{k} \sigma}^{n^{\dagger}} \hat{a}_{\mathbf{k} \sigma}^{n}+\sum_{\alpha \beta} \sum_{\mathbf{k} \mathbf{k}^{\prime} \mathbf{p}} \sum_{n n^{\prime}} \sum_{m m^{\prime}} \sum_{\sigma} \frac{1}{2 N} \cdot \\
& \cdot\left[U_{\alpha \beta} C_{\alpha \sigma}^{n}(\mathbf{k})^{*} C_{\alpha \sigma}^{n^{\prime}}(\mathbf{k}+\mathbf{p}) C_{\beta-\sigma}^{m}\left(\mathbf{k}^{\prime}\right)^{*} C_{\beta-\sigma}^{m^{\prime}}\left(\mathbf{k}^{\prime}-\mathbf{p}\right) \hat{a}_{\mathbf{k} \sigma}^{n \dagger} \hat{a}_{\mathbf{k}+\mathbf{p} \sigma}^{n^{\prime}} \hat{a}_{\mathbf{k}^{\prime}-\sigma}^{m \dagger} \hat{a}_{\mathbf{k}^{\prime}-\mathbf{p}-\sigma}^{m^{\prime}}\right. \\
+ & \left.\left(U_{\alpha \beta}-J_{\alpha \beta}\right) C_{\alpha \sigma}^{n}(\mathbf{k})^{*} C_{\alpha \sigma}^{n^{\prime}}(\mathbf{k}+\mathbf{p}) C_{\beta \sigma}^{m}\left(\mathbf{k}^{\prime}\right)^{*} C_{\beta \sigma}^{m^{\prime}}\left(\mathbf{k}^{\prime}-\mathbf{p}\right) \hat{a}_{\mathbf{k} \sigma}^{n \dagger} \hat{a}_{\mathbf{k}+\mathbf{p} \sigma}^{n^{\prime}} \hat{a}_{\mathbf{k}^{\prime} \sigma}^{m \dagger} \hat{a}_{\mathbf{k}^{\prime}-\mathbf{p} \sigma}^{m^{\prime}}\right]
\end{aligned}
$$

Now $\epsilon_{\mathbf{k} n \sigma}^{H}$ includes also the kinetic part of the single-particle hamiltonian and the Coulomb and exchange integrals are assumed to be site-independent. In the same way $H^{M F}$ becomes

$$
\hat{H}^{M F}=\sum_{\mathbf{k} n \sigma} \epsilon_{\mathbf{k} n \sigma}^{M F} \hat{a}_{\mathbf{k} \sigma}^{n^{\dagger}} \hat{a}_{\mathbf{k} \sigma}^{n}
$$

with

$$
\begin{gathered}
\epsilon_{\mathbf{k} n \sigma}^{M F}=\epsilon_{\mathbf{k} n \sigma}^{H}+Q_{\mathbf{k} \sigma}^{n} \\
Q_{\mathbf{k} \sigma}^{n}=\sum_{\alpha \beta}\left|C_{\alpha \sigma}^{n}(\mathbf{k})\right|^{2}\left[U_{\alpha \beta} \frac{1}{N} \sum_{\mathbf{k}^{\prime} n^{\prime}}^{o c c}\left|C_{\beta-\sigma}^{n^{\prime}}\left(\mathbf{k}^{\prime}\right)\right|^{2}+\left(U_{\alpha \beta}-J_{\alpha \beta}\right) \frac{1}{N} \sum_{\mathbf{k}^{\prime} n^{\prime}}^{o c c}\left|C_{\beta \sigma}^{n^{\prime}}\left(\mathbf{k}^{\prime}\right)\right|^{2}\right]
\end{gathered}
$$

which is the analogue of eq. (2.4) for Bloch states. Notice that the sums over $n^{\prime}$ are over occupied states. Equations (2.8,2.9) contain the correct recipe to include Hubbard correlation starting from band structure eigenvalues $\epsilon_{\mathbf{k} n \sigma}^{M F}$ and are essential in order to avoid double counting of e-e interaction. 


\section{HOLE SPECTRAL FUNCTION, SELF-ENERGY AND THE FADDEEV METHOD}

We are interested in the hole spectral function

$$
D_{\mathbf{k} \sigma}^{-}(\omega)=-\frac{1}{\pi} \sum_{n} \operatorname{Im}^{-}(\mathbf{k} n \sigma, \omega)
$$

which is the quantity directly related to the photoemission results we want to compare with. It describes the removal of one electron of wave-vector $\mathbf{k}$, band index $n$ and $\operatorname{spin} \sigma$ and is related to the hole-propagator

$$
\mathcal{G}^{-}(\mathbf{k} n \sigma, \omega)=-\left\langle\Psi_{0}\left|\hat{a}_{\mathbf{k} \sigma}^{n^{\dagger}} \hat{G}(z) \hat{a}_{\mathbf{k} \sigma}^{n}\right| \Psi_{0}\right\rangle ; \quad z=-\omega+E_{0}\left(N_{e}\right)+\mathrm{i} \delta
$$

$E_{0}\left(N_{e}\right)$ and $\left|\Psi_{0}\right\rangle$ define the ground state of the $N_{e}$ particle system and

$$
\hat{G}(z)=\frac{1}{z-\hat{H}^{H}}
$$

is the resolvent operator. The hole propagator can also be written in terms of the hole self-energy as

$$
\mathcal{G}^{-}(\mathbf{k} n \sigma, \omega)=\frac{1}{\omega-\epsilon_{\mathbf{k} n \sigma}^{M F}-\Sigma_{\mathbf{k} n \sigma}^{-}(\omega)}
$$

where $\Sigma_{\mathbf{k} n \sigma}^{-}(\omega)$ is the self-energy correction to band eigenvalues $\epsilon_{\mathbf{k} n \sigma}^{M F}$. In order to calculate $\Sigma_{\mathbf{k} n \sigma}^{-}(\omega)$ we proceed as in ref. [6] adopting a configuration-interaction scheme which consists in projecting the Hubbard hamiltonian on a set of states obtained by adding a finite number of e-h pairs to the Fermi sea, i.e. to the ground state $\mid \Phi_{0}>$ of the single-particle hamiltonian. We will adopt the 3-body scattering (3BS) approach where this expansion is truncated to include just one e-h pair: the state whith one removed electron of momentum $\mathbf{k}$ and spin $\sigma$ is expanded in terms of the basis set including 1- hole configurations and 3-particle configurations (1 hole +1 e-h pair) we will denote by $|s\rangle$ and $|t\rangle$ respectively

$$
|s\rangle \equiv \hat{a}_{\mathbf{k} n \sigma}\left|\Phi_{0}\right\rangle \quad|t\rangle \equiv \hat{a}_{\mathbf{q}_{3} n_{3} \sigma_{3}}^{\dagger} \hat{a}_{\mathbf{q}_{2} n_{2} \sigma_{2}} \hat{a}_{\mathbf{q}_{1} n_{1} \sigma_{1}}\left|\Phi_{0}\right\rangle
$$

with

$$
\mathbf{q}_{1}+\mathbf{q}_{2}-\mathbf{q}_{3}=\mathbf{k} ; \quad \sigma_{1}+\sigma_{2}-\sigma_{3}=\sigma
$$

To be consistent the basis set for the N-particle interacting system will include zero- and two-particle configurations. The ground state of the interacting $N_{e}$-particle system coincides then with the non-interacting one; this is obvious for the single-band hamiltonian discussed in ref. [6] (the state with zero and one e-h pair added coincide due to $\mathrm{k}$-vector conservation) and still holds in the present case of multi-band hamiltonian since $H^{H}$ has no off-diagonal matrix elements among 2-particle configurations. As a consequence in the 3BS approximation the hole propagator is the average of the resolvent over states $|s\rangle$.

By projecting the hamiltonian (2.6) over the complete set appropriate for the $N_{e}-1$ particle system we get an approximate expression for $\hat{H}^{H}$ appropriate to describe one-electron removal

$$
\hat{H}_{N_{e}-1}^{H} \simeq \hat{H}_{1}+\hat{H}_{3}+\hat{V}
$$

Here $\hat{H}_{1}$ is associated to one-hole configurations

$$
\hat{H}_{1}=\left\langle s\left|\hat{H}^{H}\right| s\right\rangle|s\rangle\langle s|
$$

$\hat{H}_{3}$ describes the contribution of 3-particle configurations

$$
\hat{H}_{3}=\sum_{t t^{\prime}}\left\langle t\left|\hat{H}^{H}\right| t^{\prime}\right\rangle|t\rangle\left\langle t^{\prime}\right|
$$

and $\hat{V}$ is the coupling between 1- and 3-particle states 


$$
\hat{V}=\sum_{t}\left\langle s\left|\hat{H}^{H}\right| t\right\rangle|s\rangle\langle t|+h . c .
$$

We leave the detailed expression of the matrix elements of the multi-band $\hat{H}^{H}$ to appendix A and proceed to sketch the method for the calculation of the resolvent (3.3). We define the 3-particle resolvent, that is the resolvent associated to the 3 -particle interaction

$$
\hat{F}_{3}(z)=\frac{1}{z-\hat{H}_{3}}
$$

and the Dyson equation which relates $\hat{G}(z)$ to it

$$
\hat{G}(z)=\hat{F}_{3}(z)+\hat{F}_{3}(z)\left[\hat{H}_{1}+\hat{V}\right] \hat{G}(z)
$$

It is a matter of simple algebra to show that the hole propagator can then be expressed in terms of $\hat{F}_{3}$ as

$$
\mathcal{G}^{-}(\mathbf{k} n \sigma, \omega)=-G_{s s}(z)=\frac{1}{\omega-E_{0}\left(N_{e}\right)+H_{s s}^{H}+\sum_{t t^{\prime}} F_{3 t t^{\prime}} V_{t^{\prime} s} V_{s t}}
$$

with the notation $G_{s s} \equiv\langle s|\hat{G}| s\rangle, F_{3 t t^{\prime}} \equiv\left\langle t\left|\hat{F}_{3}\right| t^{\prime}\right\rangle$ etc. Since the difference between the ground state energy of the $N_{e}$-particle system $E_{0}\left(N_{e}\right)$ and the average of $H^{H}$ over $\mid s>$ states turns out to be

$$
E_{0}\left(N_{e}\right)-H_{s s}^{H}=\epsilon_{\mathbf{k} n \sigma}^{H}+Q_{\mathbf{k} \sigma}^{n}=\epsilon_{\mathbf{k} n \sigma}^{M F}
$$

the band eigenvalues appear naturally in the denominator of the hole propagator and, comparing eq. (3.8) with (3.4), we can identify the self-energy correction to band eigenvalues we are interested in as

$$
\Sigma^{-}(\mathbf{k} n \sigma, \omega)=-\sum_{t t^{\prime}} F_{3 t t^{\prime}} V_{t^{\prime} s} V_{s t}
$$

The determination of the hole propagator is then reduced to the calculation of $F_{3 t t^{\prime}}$. This is done according to the Faddeev scattering theory [24] as described for the single band case in reference [6]. The method consists in separating the 3-body hamiltonian in diagonal and non-diagonal parts

$$
\begin{aligned}
\hat{H}_{3}^{D} & =\sum_{t}\left\langle t\left|\hat{H}^{H}\right| t\right\rangle|t\rangle\langle t| \\
\hat{H}_{3}^{N D} & =\sum_{t t^{\prime}}\left\langle t\left|\hat{H}^{H}\right| t^{\prime}\right\rangle|t\rangle\left\langle t^{\prime}\right|
\end{aligned}
$$

defining the diagonal 3-body resolvent

$$
\hat{F}_{3}^{D}(z)=\frac{1}{z-\hat{H}_{3}^{D}}
$$

and the scattering operator

$$
\hat{S}=\hat{H}_{3}^{N D}+\hat{H}_{3}^{N D} \hat{F}_{3}^{D} \hat{S}
$$

The 3-body resolvent can then be written as

$$
\hat{F}_{3}=\hat{F}_{3}^{D}+\hat{F}_{3}^{D} \hat{S} \hat{F}_{3}^{D}
$$

As shown in Appendix A and reference [6] the non-diagonal 3-body interaction is the sum of two potentials

$$
\hat{H}_{3}^{N D}=\hat{V}_{h-h}+\hat{V}_{h-e}
$$

which describe h-h and h-e multiple scattering.

We define partial scattering operators 


$$
\begin{aligned}
\hat{S}_{h-h} & =\hat{V}_{h-h}+\hat{H}_{h-h} \hat{F}_{3}^{D} \hat{S} \\
\hat{S}_{h-e} & =\hat{V}_{h-e}+\hat{H}_{h-e} \hat{F}_{3}^{D} \hat{S}
\end{aligned}
$$

which are related to the scattering T-matrices

$$
\begin{aligned}
& \hat{T}_{h-h}=\hat{V}_{h-h}+\hat{V}_{h-h} \hat{F}_{3}^{D} \hat{T}_{h-h} \\
& \hat{T}_{h-e}=\hat{V}_{h-e}+\hat{V}_{h-e} \hat{F}_{3}^{D} \hat{T}_{h-e}
\end{aligned}
$$

through

$$
\begin{gathered}
\hat{S}_{h-h}=\hat{T}_{h-h}+\hat{T}_{h-h} \hat{F}_{3}^{D} \hat{S}_{h-e} \\
\hat{S}_{h-e}=\hat{T}_{h-e}+\hat{T}_{h-e} \hat{F}_{3}^{D} \hat{S}_{h-h}
\end{gathered}
$$

These are the Faddeev equations which must be solved in order to get $\hat{S}=\hat{S}_{h-h}+\hat{S}_{h-e}$, $\hat{F}_{3}$ from 3.10 ) and $\mathcal{G}^{-}(\mathbf{k} n \sigma, \omega)$ from (3.8). Inserting (3.12) into (3.10) one gets the expression for the 3 - particle resolvent in terms of scattering operators $S_{h-e}$ and $T_{h-h}$

$$
\hat{F}_{3}=\hat{F}_{3}^{D}+\hat{F}_{3}^{D}\left(\hat{T}_{h-h}+\hat{T}_{h-h} \hat{F}_{3}^{D} \hat{S}_{h-e}+S_{h-e}\right) \hat{F}_{3}^{D}
$$

Further steps are necessary in order to make this general method practical for real calculations - and further approximations as well. In the following we will specialize to valence and core states.

\section{VALENCE STATES}

We adopt some semplifying assumptions for the description of photoemission from valence states of nickel. We will consider e-e correlation among $d$ electrons only and neglect the orbital dependence of one-center integrals involving valence $d$ electrons; we put

$$
\begin{array}{rlrl}
U_{\alpha \beta} & =U_{v v} & \text { for } \alpha, \beta=d \text { orbitals } \\
& =0 \quad \text { elsewhere }
\end{array}
$$

and similarly for the exchange parameter $J_{\alpha \beta}$; moreover we will neglect $U_{v v}-J_{v v}$ with respect to $U_{v v}$. In this way the hamiltonian 2.6 reduces to

$$
\begin{aligned}
\hat{H}^{H}= & \sum_{\mathbf{k} n \sigma} \epsilon_{\mathbf{k} n \sigma}^{H} \hat{a}_{\mathbf{k} \sigma}^{n^{\dagger}} \hat{a}_{\mathbf{k} \sigma}^{n}+\sum_{\alpha \beta} U_{\alpha \beta} \sum_{\mathbf{k} \mathbf{k}^{\prime} \mathbf{p}} \sum_{n n^{\prime}} \sum_{m m^{\prime}} \sum_{\sigma} \frac{1}{2 N} \cdot \\
& \cdot C_{\alpha \sigma}^{n}(\mathbf{k})^{*} C_{\alpha \sigma}^{n^{\prime}}(\mathbf{k}+\mathbf{p}) C_{\beta-\sigma}^{m}\left(\mathbf{k}^{\prime}\right)^{*} C_{\beta-\sigma}^{m^{\prime}}\left(\mathbf{k}^{\prime}-\mathbf{p}\right) \hat{a}_{\mathbf{k} \sigma}^{n \dagger} \hat{a}_{\mathbf{k}+\mathbf{p} \sigma}^{n^{\prime}} \hat{a}_{\mathbf{k}^{\prime}-\sigma}^{m \dagger} \hat{a}_{\mathbf{k}^{\prime}-\mathbf{p}-\sigma}^{m^{\prime}}
\end{aligned}
$$

Finally we will exclude configurations with e-h pair added to the majority-spin band as it would be strictly correct in the strong ferromagnetic limit where no empty states are available in the majority-spin band.

The states which define the basis set for the $N_{e}-1$ system are schematically illustrated in fig. 1; the non-zero interaction potentials responsible of $h-h$ and $e-h$ scattering are also indicated. Notice that holes and electrons of parallel spin do no interact due to the assumption $U_{v v}-J_{v v} \simeq 0$.

We have already stressed that the Hubbard correlation enters the definition of quasiparticle energies twice: first as a mean-field correction to bare eigenvalues, transforming them into band ones according to eq. (2.8); second through the addition of self-energy (3.9). The calculation of this last quantity requires a generalization of the method illustrated in detail in ref. [6] for the much simpler case of a single-band hamiltonian. The situation here is complicated by the sums over orbital indices appearing in the effective hamiltonian. This requires the definition of orbital-dependent diagonal Green functions and T-matrices as described in appendix B. As a result the self-energy correction to a band eigenvalue of wavevector $\mathbf{k}$ spin $\sigma$ and band index $n$ turns out to depend on the quantum numbers $n$ and $\mathbf{k}$ as

$$
\Sigma_{\mathbf{k} n \sigma}^{-}(\omega)=\sum_{\beta}\left|C_{\beta \sigma}^{n}(\mathbf{k})\right|^{2}\left[\sum_{\alpha} U_{\alpha \beta} \frac{1}{N} \sum_{\mathbf{k}^{\prime} \mathbf{n}^{\prime}}^{\text {empty }}\left|C_{\alpha-\sigma}^{n^{\prime}}\left(\mathbf{k}^{\prime}\right)\right|^{2}-\Sigma_{\beta \sigma}(\omega)\right]
$$


The k-vector and band-index dependence of self-energy is associated to the local orbital coefficients which modulate an orbital self-energy

$$
\Sigma_{\beta \sigma}^{-}(\omega)=\sum_{\alpha} \int_{E_{f}}^{\infty} d \epsilon n_{\alpha-\sigma}(\epsilon) T_{h-h}^{\alpha \beta}(\omega-\epsilon)\left[1+U_{\alpha \beta} A^{\alpha \beta}(\omega-\epsilon)\right]
$$

where $n_{\alpha \sigma}(\epsilon)$ is the spin-dependent orbital density of $d$ valence states and $T_{h-h}^{\alpha \beta}$ is the orbital dependent t-matrix describing the hole-hole multiple scattering

$$
T_{h-h}^{\alpha \beta}(\omega)=\frac{U_{\alpha \beta}}{1+U_{\alpha \beta} g_{3}^{\alpha \beta}(\omega)}
$$

with

$$
g_{3}^{\alpha \beta}(\omega)=\int_{-\infty}^{E_{f}} d \epsilon^{\prime} \int_{-\infty}^{E_{f}} d \epsilon \frac{n_{\alpha-\sigma}(\epsilon) n_{\beta}\left(\epsilon^{\prime}\right)}{\omega-\epsilon^{\prime}-\epsilon-i \delta}
$$

$A^{\alpha \beta}$ includes the hole-electron scattering; it is determined by solving the integral equation

$$
A^{\alpha \beta}(\omega, \epsilon)=B^{\alpha \beta}(\omega, \epsilon)+\int_{E_{f}}^{\infty} d \epsilon^{\prime} n_{\alpha-\sigma}\left(\epsilon^{\prime}\right) K^{\alpha \beta}\left(\omega, \epsilon, \epsilon^{\prime}\right) A^{\alpha \beta}\left(\omega, \epsilon^{\prime}\right)
$$

where

$$
\begin{gathered}
K^{\alpha \beta}\left(\omega, \epsilon, \epsilon^{\prime}\right)=\int_{-\infty}^{E_{f}} d \epsilon^{\prime \prime} n_{\alpha-\sigma}\left(\epsilon^{\prime \prime}\right) g_{2}^{\beta}\left(\omega+\epsilon^{\prime \prime}-\epsilon\right) T_{h-e}^{\alpha \beta}\left(\omega+\epsilon^{\prime \prime}\right) g_{2}^{\beta}\left(\omega+\epsilon^{\prime \prime}-\epsilon^{\prime}\right) T_{h-h}^{\alpha \beta}\left(\omega-\epsilon^{\prime \prime}\right) \\
B^{\alpha \beta}(\omega, \epsilon)=\int_{-\infty}^{E_{f}} d \epsilon^{\prime} n_{\alpha-\sigma}\left(\epsilon^{\prime}\right) g_{2}^{\beta}\left(\omega+\epsilon^{\prime}-\epsilon\right) T_{h-e}^{\alpha \beta}\left(\omega+\epsilon^{\prime}\right) \times \\
{\left[g_{1}^{\alpha \beta}\left(\omega-\epsilon^{\prime}\right)+\int_{E_{f}}^{\infty} d \epsilon^{\prime \prime} n_{\alpha-\sigma}\left(\epsilon^{\prime \prime}\right) g_{2}^{\beta}\left(\omega+\epsilon^{\prime}-\epsilon^{\prime \prime}\right) g_{3}^{\alpha \beta}\left(\omega-\epsilon^{\prime \prime}\right) T_{h-h}^{\alpha \beta}\left(\omega-\epsilon^{\prime \prime}\right)\right]}
\end{gathered}
$$

$T_{h-e}^{\alpha \beta}$ is the orbital dependent t-matrix describing the hole-electron scattering

$$
T_{h-e}^{\alpha \beta}(\omega)=\frac{-U_{\alpha \beta}}{1-U_{\alpha \beta} g_{1}^{\alpha \beta}(\omega)}
$$

with

$$
g_{1}^{\alpha \beta}(\omega)=\int_{-\infty}^{E_{f}} d \epsilon^{\prime} \int_{E_{f}}^{\infty} d \epsilon \frac{n_{\alpha-\sigma}(\epsilon) n_{\beta \sigma}\left(\epsilon^{\prime}\right)}{\omega-\epsilon^{\prime}+\epsilon-i \delta}
$$

and finally

$$
g_{2}^{\beta}(\omega)=\int_{-\infty}^{E_{f}} d \epsilon^{\prime} \frac{n_{\beta \sigma}\left(\epsilon^{\prime}\right)}{\omega-\epsilon^{\prime}-i \delta}
$$

Equations (4.2 - 4.11) describe the procedure we have followed to calculate in practice self-energy corrections for the valence states of $\mathrm{Ni}$ reported in sec. VI.

\section{CORE STATES}

The localized character of core states is responsible for much stronger correlation effects associated with larger on-site Coulomb and exchange integrals. In order to adapt the hamiltonian (2.6) to the case of core states we make the following assumptions: we neglect the correlation among valence electrons and assume them to be described by a 
single band. In 2.6 the band index $n$ can have just two values and as does the orbital index and we are left with a two-band hamiltonian

$$
\hat{H}^{H}=\hat{H}_{v v}+\hat{H}_{c c}+\hat{H}_{c v}
$$

where $\hat{H}_{v v}$ is the band hamiltonian for valence electrons with the e-e interaction treated in the mean-field approximation

$$
\hat{H}_{v v}=\sum_{\mathbf{k} \sigma} \epsilon_{\mathbf{k} v \sigma}^{M F} \hat{a}_{\mathbf{k} v \sigma}^{\dagger} \hat{a}_{\mathbf{k} v \sigma}
$$

and

$$
\begin{aligned}
& \hat{H}_{c c}=\sum_{\mathbf{k} \sigma} \epsilon_{c \sigma}^{H} \hat{a}_{\mathbf{k} c \sigma}^{\dagger} \hat{a}_{\mathbf{k} c \sigma}+\sum_{\mathbf{k} \mathbf{k}^{\prime} \mathbf{p}} \sum_{\sigma} \frac{U_{c c}}{N} \hat{a}_{\mathbf{k} c \sigma}^{\dagger} \hat{a}_{\mathbf{k}+\mathbf{p} c \sigma} \hat{a}_{\mathbf{k}^{\prime} c-\sigma}^{\dagger} \hat{a}_{\mathbf{k}^{\prime}-\mathbf{p} c-\sigma} \\
\hat{H}_{c v} & =\sum_{\mathbf{k} \mathbf{k}^{\prime} \mathbf{p}} \sum_{\sigma} \frac{1}{N}\left[U_{c v} C_{v-\sigma}\left(\mathbf{k}^{\prime}\right)^{*} C_{v-\sigma}\left(\mathbf{k}^{\prime}-\mathbf{p}\right) \hat{a}_{\mathbf{k} c \sigma}^{\dagger} \hat{a}_{\mathbf{k}+\mathbf{p} c \sigma} \hat{a}_{\mathbf{k}^{\prime} v-\sigma}^{\dagger} \hat{a}_{\mathbf{k}^{\prime}-\mathbf{p} v-\sigma}\right. \\
& \left.+\left(U_{c v}-J_{c v}\right) C_{v \sigma}\left(\mathbf{k}^{\prime}\right)^{*} C_{v \sigma}\left(\mathbf{k}^{\prime}-\mathbf{p}\right) \hat{a}_{\mathbf{k} c \sigma}^{\dagger} \hat{a}_{\mathbf{k}+\mathbf{p} c \sigma} \hat{a}_{\mathbf{k}^{\prime} v \sigma}^{\dagger} \hat{a}_{\mathbf{k}^{\prime}-\mathbf{p} v \sigma}\right]
\end{aligned}
$$

Here $U_{c c}=J_{c c}, U_{c v}$, and $J_{c v}$ describe the interactions between core - core and core - valence $d$ electrons.

Figure 2 shows schematically the configurations to be included for the 3BS description. Notice that configurations with one e-h pair added to the majority-spin band are now considered: even if the number of empty states available is small, the stronger value of the interactions makes these scattering channels no more negligible. As shown in fig. 2 we have then to take into account scattering between particles of parallel spin proportional to $U_{c v}-J_{c v}$ and between particles of opposite spin proportional to $U_{c v}$. The extra configurations where all the particles (holes and electrons) have the same spin are called $\mid z>$.

The procedure to calculate self-energy for core states is a straightforward extension of the one outlined for valence states. The core hole propagator turns out to be given by

$$
\mathcal{G}^{-}(\mathbf{k} c \sigma, \omega)=\frac{1}{\omega-\epsilon_{c \sigma}^{M F}-\sum_{t t^{\prime}} F_{3 t t^{\prime}} V_{t^{\prime} s} V_{s t}-\sum_{z z^{\prime}} F_{3 z z^{\prime}} V_{z^{\prime} s} V_{s z}}
$$

The presence here of extra configurations and extra interactions does not imply any major difference with respect to the case of valence states - just the addition of an extra term in the denominator of (5.2) and the necessity to solve separately two Faddeev problems to calculate $F_{3 t t^{\prime}}$ and $F_{3 z z^{\prime}}$ for opposite and parallel spin interactions respectively. This is a consequence of the fact that the hamiltonian (5.1) does not mix $\mid z>$ and $\mid t>$ configurations. The Faddeev problem for the determination of $F_{3 z z^{\prime}}$ is solved in the same way as described in appendix B for $F_{3 t t^{\prime}}$, substituting $U_{c v}$ with $U_{c v}-J_{c v}$ and parallel spin instead of opposite ones. Moreover the description of the core state in terms of a zero-width band with a delta function as orbital density of states drastically reduces the computational effort required to evaluate the non-interacting Green functions 4.5, 4.10, 4.11).

The relationship between the energy $\epsilon_{c \sigma}^{M F}$ and the bare core eigenvalue involves as usual $Q_{\mathbf{k} \sigma}^{n}$ (see eq. 2.8 and 2.9 ); explicitly one has

$$
\epsilon_{c \sigma}^{M F}=\epsilon_{c \sigma}^{H}+\left[\left(U_{c v}-J_{c v}\right)<\hat{n}_{v \sigma}>+U_{c v}<\hat{n}_{v-\sigma}>\right]+U_{c c}<\hat{n}_{c-\sigma}>
$$

It is interesting to make a comment concerning the role of the Coulomb repulsion $U_{c c}$. Since any 3 -particle $(1$ hole plus one e-h pair) configuration must involve empty states, no multiple scattering is associated with $U_{c c}$ and this quantity gives rise just to a mean field contribution. Core-valence interaction $U_{c v}$ and $J_{c v}$ on the contrary modify the bare core energies both through the mean field contribution and by self-energy corrections which originate from hole-hole and electron-hole scattering of opposite and parallel spin described by $F_{3 t t^{\prime}}$ and $F_{3 z z^{\prime}}$ respectively.

\section{RESULTS}

The calculation of self-energy corrections requires as an input i) the mean-field eigenvalues and eigenvectors for valence electrons, ii)the energy of the core level and iii) the values of the Coulomb and exchange parameters $U_{v v}$, 
$U_{c c}, U_{c v}, J_{c v}$. All these quantities can be deduced from an ab-initio LDA calculation; Coulomb integrals in particular can be obtained in the so-called constrained-density functional scheme [25] - a procedure which is however not free of ambiguites (see for instance the discussion reported in ref. [13]) and can lead to large variations in the estimated values. We have therefore adopted a mixed strategy, using results of ab-initio band calculations to get quantities i) only, treating all the others as free parameters.

The band structure of ferromagnetic nickel has been calculated with the linear muffin-tin orbital (LMTO) method in the atomic-spheres approximation (ASA) including the combined correction term [26]. The tight-binding LMTO basis set 27 has been used, including 9 orbitals $(s, p d)$ per atom. The resulting energy dispersion is shown in fig. 3 ; the occupation numbers for valence $d$ orbitals turns out to be $\left\langle n_{v \uparrow}\right\rangle=4.68,\left\langle n_{v \downarrow}\right\rangle=4.07$. We have used the single-particle eigenvalues and the corresponding $d$ contribution to eigenfunctions and orbital densities of states to calculate self-energy corrections and spectral functions according to the theory described in the previous sections. A value of $U_{v v}=2 \mathrm{eV}$ has been chosen in order to reproduce the observed energy position of the valence band satellite; as we will show below we are able in this way to reproduce also other characteristics of the valence quasi-particle states such as band width, quasi-particle energy dispersion and exchange splitting; this is an important result and represents a success of the present approach: previous methods based on a semplified description of the scattering channels [14.15] in fact have not been able to reproduce at the same time the satellite energy position and the valence band width which turned out to be systematically overestimated for values of the Coulomb integral fixed to reproduce the satellite binding energy.

Fig. 4 shows the comparison between our results and recent angle-integrated / spin-resolved photoemission data [3]. We find that the calculated total spectral function $D_{\sigma}^{-}(\omega)$ defined as

$$
D_{\sigma}^{-}(\omega)=\sum_{\mathbf{k} n} D_{\mathbf{k} n \sigma}^{-}(\omega)
$$

closely reproduces the experimental energy distribution curves for each spin component; notice in particular that the $6 \mathrm{eV}$ satellite is observed clearly only in the photoemission from majority-spin states in agreement with our results. The two approximations we have adopted for the description of valence states, that is $U_{v v}-J_{v v} \simeq 0$ and the strong ferromagnetic limit make the self-energy corrections exactly zero for minority-spin bands and the comparison between our results and the spin-resolved experimental data confirms the validity of both these assumptions. As a further evidence of this we report in fig. 4 the minority-spin spectral function calculated after removing the assumption of strong ferromagnetism, i.e. considering also e-h pairs added to the majority-spin band; the small number of empty states and the relatively small value of the interaction $U_{v v}$ make these scattering channels not efficient and the calculated spectrum is not significantly altered.

It is possible to perform a more refined analysis by looking at the k- resolved spectral function; fig. 5 (a) shows the spectral function for the $K$ point and $\epsilon_{\mathbf{k} n \uparrow}^{M F}=-3.6 \mathrm{eV}$ together with the corresponding self-energy $\Sigma_{\mathbf{k} n \uparrow}^{-}(\omega)$. The spectral function shows a quasi-particle peak plus a satellite: the first structure is associated to the pole of the hole propagator shown in fig. 5 (b) as the interception between $\operatorname{Re}\left(\Sigma_{\mathbf{k} n \uparrow}^{-}(\omega)\right)$ and the straight line $\omega-\epsilon_{\mathbf{k} n \uparrow}^{M F}$. The second structure is associated to the maximum of $\operatorname{Im}\left(\Sigma_{\mathbf{k} n \uparrow}^{-}(\omega)\right)$ and as such it will occur at the same energy at any $\mathrm{k}$-vector. This appears more clearly by extending the same analysis to the k-points along the high symmetry line of the Brillouin Zone and plotting the energy position of the maxima of $D_{\mathbf{k} \sigma}^{-}(\omega)=\sum_{n} D_{\mathbf{k} n \sigma}^{-}(\omega)$ to get the quasi-particle band structure of fig. 6. By comparing the quasi-particle band structure with the single-particle results it appears that the majority-spin eigenvalues are heavily affected by self-energy corrections, showing a strong reduction of the $d$ - band width and the presence of the above mentioned $6 \mathrm{eV}$ satellite. Since the majority-spin eigenvalues are shifted to lower binding energies while the minority-spin ones are unaffected, the splitting between majority and minority states becomes smaller than in the original single particle bands. This goes in the right direction since it is well known that single particle calculations overestimate this quantity; from fig. 6 it appears that the energy separation between majority and minority quasiparticle peacks around $\Gamma$ point is $0.2 \mathrm{eV}$ for the topmost band. This result is in remarkable agreement with a recent estimate reporting a split of $204 \pm 8 \mathrm{meV}$ along the $\Sigma$ direction 28]. The same is true for the quasi-particle energy dispersion as a whole, which is shown in fig. 7 compared with the results of angle-resolved spin-integrated photoemission spectroscopy of ref. [29].

As far as core levels are concerned we consider here the $3 p$ level of nickel as a test case. Since the core levels of an isolated atom are degenerate in spin their spin dependence is a solid state effect, associated to the interaction between the atom and the solid it is embedded in; in other words the spin dependence of core level energies is related to the spin polarization of the valence band on one side and to core-valence interactions on the other. According to our view the relevant quantities are the Coulomb and exchange integrals between core and valence orbitals which affect the bare atomic core energies both through the mean field term $Q_{\mathbf{k} \sigma}^{n}$ and self-energy corrections. The mean-field contribution of eq. (5.3) gives rise to a spin splitting proportional to $J_{c v}$ and to the valence band spin polarization 


$$
\epsilon_{c \uparrow}^{M F}-\epsilon_{c \downarrow}^{M F}=J_{c v}\left(<\hat{n}_{v \downarrow}>-<\hat{n}_{v \uparrow}>\right)
$$

Notice that $U_{c v}$ and $U_{c c}$ do not enter this expression; they affect the bare core level $\epsilon_{c \uparrow}^{H}=\epsilon_{c \downarrow}^{H}$ according to

$$
\epsilon_{c \uparrow}^{H}+\left[U_{c v}\left(<\hat{n}_{v \uparrow}>+<\hat{n}_{v \downarrow}>\right)+U_{c c}<\hat{n}_{c \downarrow}>\right] \equiv \epsilon_{c}
$$

giving rise to a modified core energy level $\epsilon_{c}$ which is still spin-independent. The mean-field eigenvalues are related to this quantity as

$$
\epsilon_{c \sigma}^{M F}=\epsilon_{c}-J_{c v}<\hat{n}_{v \sigma}>
$$

We have used spin-orbit split values of $\epsilon_{c}$, that is $\epsilon_{c}^{3 / 2}$ and $\epsilon_{c}^{1 / 2}$, to fix the absolute value of the core level binding energy, choosing then $J_{c v}$ to reproduce the spin-splitting of the main peak observed in the core-level photoemssion [3]. The last parameter, that is $U_{c v}$ has been fixed in order to reproduce the satellites energy position. The values which optimize the agreement between our calculation and the experimental results are listed in Table [

Fig. 8 shows the calculated self-energy for the creation of both majority and minority-spin hole in the core level $3 p^{1 / 2}$. The same analysis obviously applies to the other spin-orbit split level $3 p^{3 / 2}$. In the case of the minority-spin core hole the self-energy presents two well defined structures: the one at lower binding energy is related to the scattering between particles of parallel spin with strength proportional to $U_{c v}-J_{c v}$ (see fig. 2(f)) while the structure at higher binding energy is related to $U_{c v}$ and to the scattering between opposite spin particles (see fig. 2 (e)). Notice that two independent factors determine the efficiency of the scattering process, the strength of the interaction on one side and the number of available states in the valence band on the other. In the case of a minority-spin core hole the weaker parallel spin interaction is compensated by the larger number of empty valence states and both the scattering channels involving parallel and opposite spin particles play a role. The same argument applies to the case of majority-spin core hole but now the weaker parallel spin interaction is associated to a small number of available empty valence states (see fig. 2 c); for this reason the interaction between opposite spin particles (see fig. 2 (b)) remains the only efficient scattering channel. As a consequence the self-energy for majority-spin core hole presents a single structure associated to $U_{c v}$.

As discussed in the previous section satellite structures are expected to occur at energies where the imaginary part of self-energy has a maximum giving rise to a complex pole of the hole propagator and therefore to a short lived excitation. The structures in the calculated self-energy we have just described and their origin are therefore essential in order to get a physical interpretation of the observed photoemission spectrum. We report in fig. 9 the calculated spin-integrated spectral density for the creation of a $3 p$ core hole compared with the photoemission results of ref. [22]. The spectrum shows a main peak at about $66 \mathrm{eV}$ with two characteristic spin-orbit split structures (A, B) and two satellites $(\mathrm{C}, \mathrm{D})$. The decomposition of the spectral function into contributions from the two spin-orbit split levels $3 p^{1 / 2}$ and $3 p^{3 / 2}$ and from different spins is also shown. The spectral function for the creation of a majority-spin core hole shows a main peack and a satellite for each spin-orbit split level; as discussed above in terms of the selfenergy this satellite is associated to the only efficient scattering channel which comes into play after the removal of one majority- spin electron, that is to configurations of fig. 2. (b) where the majority-spin core hole interacts with opposite spin particles in the valence band, with a strength proportional to $U_{c v}$. The spectral function for the creation of a minority-spin core hole presents instead two satellites for each spin-orbit split level: the one at the higher binding energy is associated to the configurations of fig. 2 (e) where the spin-down core hole interacts with opposite spin particles in the valence band with a strength proportianal to $U_{c v}$. Due to the small number of empty states available in the spin-up band this satellites is less pronounced here than in the majority-spin spectrum. The satellite at lower binding energy is related to configurations of fig. 2 (f) and to scattering between parallel spin particles of strenght proportional to $U_{c v}-J_{c v}$.

The one-to-one correspondence between the configurations of fig. 2 and the satellite structures allows us to interpret them as as shake-up processes occurring after the removal of either a minority or a majority-spin core electron. The satellite at lower binding energy (C) is then associated to the creation of one minority-spin core-hole plus an e-h pair in the valence band of the same spin, giving rise to a bound state of three parallel spin particles which can be defined AS a triplet state; the satellite at higher binding energy (D) is related to the creation of either a majority or a minority-spin core hole plus a valence e-h pair of opposite spin giving rise to a singlet state.

To analyze the spin polarization of the whole core hole spectrum in more details it is useful to consider the spinresolved spectra and their difference $D_{\uparrow}^{-}(\omega)-D_{\downarrow}^{-}(\omega)$ reported in fig. 10. It appears that the spin polarity of structures A, B, C and D of fig. 9 is "down", "up", "down", "up" respectively. This is again in agreement with what has been seen experimentally and reported in ref. [19,22, 23]. 


\section{SUMMARY}

We have described a method for including short-range on-site interactions in the description of both valence and core states of a solid system. When applied to valence states of ferromagnetic nickel the method allows to get a quasiparticle band structure which compares much more favourably with the experimental observation than conventional meanfield LDA band structure eigenvalues, reproducing the observed band width, the energy dispersion, the satellite structure and the exchange splitting. Since the method does not rely on a perturbation expansion it has a wide range of application, including any correlation regime. The extension to core levels is quite natural and allows to take fully into account the itinerant character of valence electrons. We get then a physical picture of the $3 p$ core level spectrum where the spin splitting of the main line is associated to the valence band spin polarization and to the core-valence exchange interaction; the two satellites are interpreted as arising from shake-up processes occurring after the removal of a core electron, involving the creation of a $d$ band e-h pair. Even though our present choice of empirically determining the parameters of the Hubbard hamiltonian ensures that we obtain an overall good agreement with experiments we believe that the possibility of reproducing both the satellite structures, the main line and their spin dependence with just four parameters can be seen as a non trivial result. The widely used atomic models - which use empirical parameters as well - require also an ad-hoc evaluation of the so called extra-atomic effects which allow to take into account the role of electrons on neighbouring atoms 21]; such effects are on the contrary included here from the beginning since the full details of the valence band structure of the solid system are considered. In this sense the present approach can be considered an appropriate tool to describe the response of an itinerant electron system to the creation of a core hole.

\section{APPENDIX A: MATRIX ELEMENTS OF THE MULTI-ORBITAL HUBBARD HAMILTONIAN}

As discussed in section IV the application of the 3BS method to the valence band states of nickel requires the explicit definition of matrix elements of the Hamiltonian 4.1 containing a non-interacting part that we call here $\hat{H}^{0}$ and the on-site interaction among opposite spin electrons that we denote by $\hat{H}^{\prime}$. The matrix elements involve states $\mid s>$ and $\mid t>$ defined in 3.5 and pictorially depicted in fig. 1 . We have

$$
\begin{gathered}
\left\langle s\left|\hat{H}^{0}\right| s\right\rangle=\sum_{k^{\prime} n^{\prime} \sigma^{\prime}}^{o c c} \epsilon_{k^{\prime} n^{\prime} \sigma^{\prime}}^{H}-\epsilon_{k n \sigma}^{H} \\
\left\langle s\left|\hat{H}^{0}\right| t\right\rangle=0 \\
\left\langle t\left|\hat{H}^{0}\right| t\right\rangle=\sum_{k^{\prime} n^{\prime} \sigma^{\prime}}^{o c c} \epsilon_{k^{\prime} n^{\prime} \sigma^{\prime}}^{H}+\epsilon_{q_{3} n_{3}-\sigma}^{H}-\epsilon_{q_{2} n_{2}-\sigma}^{H}-\epsilon_{q_{1} n_{1} \sigma}^{H} \\
\left\langle s\left|\hat{H}^{\prime}\right| s\right\rangle=\sum_{\alpha \beta} \frac{U_{\alpha \beta}}{N} \sum_{k^{\prime \prime} n^{\prime \prime}}\left|C_{\alpha-\sigma}^{n^{\prime \prime}}\left(\boldsymbol{k}^{\prime \prime}\right)\right|^{2}\left\{\sum_{k^{\prime} n^{\prime}}\left|C_{\beta \sigma}^{n^{\prime}}\left(\boldsymbol{k}^{\prime}\right)\right|^{2}-\left|C_{\beta \sigma}^{n}(\boldsymbol{k})\right|^{2}\right\} \\
\left\langle s\left|\hat{H}^{\prime}\right| t\right\rangle=-\sum_{\alpha \beta} \frac{U_{\alpha \beta}}{N} C_{\alpha-\sigma}^{n_{2}}\left(\boldsymbol{q}_{2}\right)^{*} C_{\alpha-\sigma}^{n_{3}}\left(\boldsymbol{q}_{3}\right) C_{\beta \sigma}^{n_{1}}\left(\boldsymbol{q}_{1}\right)^{*} C_{\beta \sigma}^{n}(\boldsymbol{k}) \delta_{k-q_{1}, q_{2}-q_{3}} \\
\left\langle t\left|\hat{H}^{\prime}\right| t\right\rangle=\sum_{\alpha \beta} \frac{U_{\alpha \beta}}{N}\left\{\sum_{k^{\prime} n^{\prime}}\left|C_{\alpha-\sigma}^{n^{\prime}}\left(\boldsymbol{k}^{\prime}\right)\right|^{2} \sum_{k^{\prime \prime} n^{\prime \prime}}\left|C_{\beta \sigma}^{n^{\prime \prime}}\left(\boldsymbol{k}^{\prime \prime}\right)\right|^{2}+\left|C_{\alpha-\sigma}^{n_{3}}\left(\boldsymbol{q}_{3}\right)\right|^{2} \sum_{k^{\prime} n^{\prime}}\left|C_{\beta \sigma}^{n^{\prime}}\left(\boldsymbol{k}^{\prime}\right)\right|^{2}+\right. \\
\left.-\left|C_{\alpha-\sigma}^{n_{2}}\left(\boldsymbol{q}_{2}\right)\right|^{2} \sum_{k^{\prime} n^{\prime}}\left|C_{\beta \sigma}^{n^{\prime}}\left(\boldsymbol{k}^{\prime}\right)\right|^{2}-\left|C_{\beta \sigma}^{n_{1}}\left(\boldsymbol{q}_{1}\right)\right|^{2} \sum_{k^{\prime} n^{\prime}}\left|C_{\alpha-\sigma}^{n^{\prime}}\left(\boldsymbol{k}^{\prime}\right)\right|^{2}\right\} \\
\left\langle t^{\prime}\left|\hat{H}^{\prime}\right| t\right\rangle=-\sum_{\alpha \beta} \frac{U_{\alpha \beta}}{N} C_{\alpha-\sigma}^{n_{3}^{\prime}}\left(\boldsymbol{q}_{3}^{\prime}\right)^{*} C_{\alpha-\sigma}^{n_{3}}\left(\boldsymbol{q}_{3}\right) C_{\beta \sigma}^{n_{1}}\left(\boldsymbol{q}_{1}\right)^{*} C_{\beta \sigma}^{n_{1}^{\prime}}\left(\boldsymbol{q}_{1}^{\prime}\right) \delta_{q_{2}, q_{2}^{\prime}} \delta_{n_{2}, n_{2}^{\prime}} \delta_{q_{1}-q_{3}, q_{1}^{\prime}-q_{3}^{\prime}}+ \\
+\sum_{\alpha \beta} \frac{U_{\alpha \beta}}{N} C_{\alpha-\sigma}^{n_{2}}\left(\boldsymbol{q}_{2}\right)^{*} C_{\alpha-\sigma}^{n_{2}^{\prime}}\left(\boldsymbol{q}_{2}^{\prime}\right) C_{\beta \sigma}^{n_{1}}\left(\boldsymbol{q}_{1}\right)^{*} C_{\beta \sigma}^{n_{1}^{\prime}}\left(\boldsymbol{q}_{1}^{\prime}\right) \delta_{q_{3}, q_{3}^{\prime}} \delta_{n_{3}, n_{3}^{\prime}} \delta_{q_{1}+q_{2}, q_{1}^{\prime}+q_{2}^{\prime}}
\end{gathered}
$$

As discussed in section $\mathrm{V}$ the description of core states requires the inclusion of the interaction involving the exchange integral that we call here $\hat{H}^{\prime \prime}$ and the extension of the complete set to the three-particle configurations $\mid z>$ 
of fig. 2. It is easy to show that in this case the diagonal matrix elements are similar to the previous ones and that the non- zero off-diagonal ones are $\left\langle s\left|\hat{H}^{\prime}\right| t\right\rangle,\left\langle t\left|\hat{H}^{\prime}\right| t^{\prime}\right\rangle,\left\langle z\left|\hat{H}^{\prime \prime}\right| z^{\prime}\right\rangle$.

\section{APPENDIX B: FADDEEV EQUATIONS FOR THE MULTI-BAND HUBBARD HAMILTONIAN}

We illustrate the procedure which leads to eq. (4.2) - (4.10) for the removal of one spin up electron. The relationship (3.8) between the diagonal hole-propagator and the 3-body resolvent is obtained by inserting the completness relation

$$
\sum_{s}|s><s|+\sum_{t}|t><t|
$$

into the identity

$$
<s\left|\left(z-\hat{H^{H}}\right) \frac{1}{z-\hat{H}^{H}}\right| s>=1
$$

that is

$$
\left[z-\left(H_{1}\right)_{s s}\right] G_{s s}(z)-\sum_{t} V_{s t} G_{t s}(z)=1
$$

Since $G_{t s}(z)$ can be obtained by eq. (3.7) as

$$
G_{t s}(z)=\sum_{t s^{\prime}} F_{t t^{\prime}} V_{t^{\prime} s^{\prime}} G_{s s^{\prime}}(z)
$$

one gets

$$
\left[z-\left(H_{1}\right)_{s s}\right] G_{s s}(z)-\sum_{t t^{\prime}} V_{s t} V_{t^{\prime} s} F_{t t^{\prime}} G_{s s}+\sum_{t t^{\prime}} \sum_{s \neq s^{\prime}} V_{s t} V_{t^{\prime} s^{\prime}} F_{t t^{\prime}} G_{s s^{\prime}}=1
$$

By substituting the explicit definition of the matrix elements of $\hat{V}$ it appears that the two summations in (B3) involve terms of the kind

$$
\sum_{\beta \delta} C_{\beta \uparrow}^{n}(\mathbf{k}) C_{\delta \uparrow}^{n^{\prime}}(\mathbf{k})^{*}
$$

with $n=n^{\prime}, n \neq n^{\prime}$ in the first and second sum respectively. For this reason it seams reasonable to neglect in (B3) the last summation with respect to the first one getting in this way eq. (3.8) as a result.

According to eq. (3.9) the self-energy is given by the sum

$$
\begin{aligned}
\sum_{t t^{\prime}} F_{3 t t^{\prime}} V_{t^{\prime} s} V_{s t}= & \frac{U^{2}}{N^{2}} \sum_{q_{1} q_{2} q_{3}} \sum_{n_{1} n_{2} n_{3}} \sum_{\alpha \beta} C_{\alpha \uparrow}^{n_{1}}\left(\mathbf{q}_{\mathbf{1}}\right)^{*} C_{\alpha \downarrow}^{n_{3}}\left(\mathbf{q}_{\mathbf{3}}\right) C_{\beta \downarrow}^{n_{2}}\left(\mathbf{q}_{\mathbf{2}}\right)^{*} C_{\beta \uparrow}^{n}(\mathbf{k}) \\
& \cdot \sum_{q_{1}^{\prime} q_{2}^{\prime} q_{3}^{\prime}} \sum_{n_{1}^{\prime} n_{2}^{\prime} n_{3}^{\prime}} \sum_{\gamma \delta} C_{\gamma \uparrow}^{n_{1}^{\prime}}\left(\mathbf{q}_{\mathbf{1}}^{\prime}\right) C_{\gamma \downarrow}^{n_{3}^{\prime}}\left(\mathbf{q}_{\mathbf{3}}^{\prime}\right)^{*} C_{\delta \downarrow}^{n_{2}^{\prime}}\left(\mathbf{q}_{\mathbf{2}}^{\prime}\right) C_{\delta \uparrow}^{n}(\mathbf{k})^{*} \\
& \cdot<q_{1} n_{1} \uparrow q_{2} n_{2} \downarrow q_{3} n_{3} \downarrow \| \hat{F}_{3}^{D}+\hat{F}_{3}^{D}\left(\hat{S}_{h-e}+\hat{T}_{h-h} \hat{F}_{3}^{D} \hat{S}_{h-e}\right) \hat{F}_{3}^{D} \\
& \cdot \mid q_{1}^{\prime} n_{1}^{\prime} \uparrow q_{2}^{\prime} n_{2}^{\prime} \downarrow q_{3}^{\prime} n_{3}^{\prime} \downarrow>
\end{aligned}
$$

where we have used the definition 3.5 for $\mid t>$ and $\left|t^{\prime}\right\rangle$. Let us define now the orbital dependent free propagator describing h-h scatterings

$$
g_{3}^{\alpha \beta}\left(\mathbf{q}_{3} n_{3} \omega\right)=\frac{1}{N^{2}} \sum_{q_{1} n_{1} q_{2} n_{2}} \frac{C_{\alpha \uparrow}^{n_{1}}\left(\mathbf{q}_{\mathbf{1}}\right)^{*} C_{\alpha \uparrow}^{n_{1}}\left(\mathbf{q}_{\mathbf{1}}\right) C_{\beta \downarrow}^{n_{2}}\left(\mathbf{q}_{2}\right)^{*} C_{\beta \downarrow}^{n_{2}}\left(\mathbf{q}_{\mathbf{2}}\right)}{\omega-\left(E_{0}-\epsilon_{\mathbf{q}_{1} n_{1} \uparrow}^{M F}-\epsilon_{\mathbf{q}_{2} n_{2} \downarrow}^{M F}+\epsilon_{\mathbf{q}_{3} n_{3} \downarrow}^{M F}\right)}
$$

where the summation is over filled states of band indeces $n_{1}$ and $n_{2}$. By using the definition (3.11) it is easy to show that 


$$
\begin{aligned}
& \sum_{q_{1} n_{1} q_{2} n_{2}} C_{\alpha \uparrow}^{n_{1}}\left(\mathbf{q}_{\mathbf{1}}\right)^{*} C_{\beta \downarrow}^{n_{2}}\left(\mathbf{q}_{\mathbf{2}}\right)^{*}<q_{1} n_{1} \uparrow q_{2} n_{2} \downarrow q_{3} n_{3} \downarrow\left|\hat{G}_{3}^{D} \hat{T}_{h-h}\right| q_{1}^{\prime} n_{1}^{\prime} \uparrow q_{2}^{\prime} n_{2}^{\prime} \downarrow q_{3}^{\prime} n_{3}^{\prime} \downarrow>= \\
= & \frac{C_{\alpha \uparrow}^{n_{1}}\left(\mathbf{q}_{\mathbf{1}}\right)^{*} C_{\beta \downarrow}^{n_{2}}\left(\mathbf{q}_{\mathbf{2}}\right)^{*} g_{3}^{\alpha \beta}\left(\mathbf{q}_{\mathbf{3}} \omega\right)}{1-U_{\alpha \beta} g_{3}^{\alpha \beta}\left(\mathbf{q}_{\mathbf{3}} \omega\right)} \delta_{\mathbf{q}_{\mathbf{3}}, \mathbf{q}_{\mathbf{3}}^{\prime}}
\end{aligned}
$$

It is also useful to define the orbital-dependent t-matrix for h-h scatterings

$$
T_{h-h}^{\alpha \beta}\left(\mathbf{q}_{3} n_{3} \omega\right)=\frac{U_{\alpha \beta}}{1-U_{\alpha \beta} g_{3}^{\alpha \beta}\left(\mathbf{q}_{3} n_{3} \omega\right)}
$$

Similar definitions and relations hold for e-h scatterings. It is then a matter of simple algebra to transform eq. (B5) into the form

$$
\begin{aligned}
\sum_{t t^{\prime}} F_{3 t t^{\prime}} V_{t^{\prime} s} V_{s t} & =\sum_{\beta}\left|C_{\beta}^{n}(\mathbf{k} \uparrow)\right|^{2} \\
& -\sum_{\alpha} \frac{U_{\alpha \beta}}{N} \sum_{\mathbf{q}_{3} n_{3}}\left[\left|C_{\beta}^{n}\left(\mathbf{q}_{\mathbf{3}} \downarrow\right)\right|^{2}\right. \\
& +T_{h-h}^{\alpha \beta}\left(\mathbf{q}_{\mathbf{3}} n_{3}\right)\left(\left|C_{\beta}^{n}\left(\mathbf{q}_{\mathbf{3}} \downarrow\right)\right|^{2}+U_{\alpha \beta} A^{\alpha \beta}\left(\mathbf{q}_{\mathbf{3}} n_{3}\right)\right)
\end{aligned}
$$

where

$$
\begin{aligned}
A^{\alpha \beta}\left(\mathbf{q}_{\mathbf{3}} n_{3}\right)= & \frac{1}{N} \sum_{q_{1} q_{2}} \sum_{n_{1} n_{2}} C_{\alpha \uparrow}^{n_{1}}\left(\mathbf{q}_{\mathbf{1}}\right)^{*} C_{\beta \downarrow}^{n_{2}}\left(\mathbf{q}_{\mathbf{2}}\right)^{*} C_{\alpha \downarrow}^{n_{3}}\left(\mathbf{q}_{\mathbf{3}}\right) \\
& \cdot \sum_{q_{1}^{\prime} q_{2}^{\prime} q_{3}^{\prime}} \sum_{n_{1}^{\prime} n_{2}^{\prime} n_{3}^{\prime}} C_{\gamma \uparrow}^{n_{1}^{\prime}}\left(\mathbf{q}_{1}^{\prime}\right) C_{\gamma \downarrow}^{n_{3}^{\prime}}\left(\mathbf{q}_{\mathbf{3}}^{\prime}\right)^{*} C_{\delta \downarrow}^{n_{2}^{\prime}}\left(\mathbf{q}_{\mathbf{2}}^{\prime}\right) \\
& \cdot<q_{1} n_{1} \uparrow q_{2} n_{2} \downarrow q_{3} n_{3} \downarrow\left|\hat{F}_{3}^{D} \hat{S}_{h-e} \hat{F}_{3}^{D}\right| q_{1}^{\prime} n_{1}^{\prime} \uparrow q_{2}^{\prime} n_{2}^{\prime} \downarrow q_{3}^{\prime} n_{3}^{\prime} \downarrow>
\end{aligned}
$$

From now on the procedure is the same as the one described in ref. [6], adopting in particular the so-called local approximation [10] which allows to transform all the summation over k-vectors into integrals involving the density of states.

[1] L. C. Davis J. Appl. Phys. 59, R25 (1986) and references therein

[2] Y. Kakehashi, K. Becker, P. Fulde, Phys. Rev. B 29, 16 (1984)

[3] A. K. See and L.E. Klebanoff , Phys. Rev. B 51, 11002 (1995)

[4] F. Manghi, C. Calandra, S. Ossicini, Phys. Rev. Lett. 73, 3129 (1994)

[5] J. Igarashi , J. Phys. Soc. Japan 522827 (1983).

[6] C. Calandra, F. Manghi , Phys. Rev. B 50, 2061 (1994)

[7] A. Villafiorita, F. Manghi, F. Parmigiani, C. Calandra, submitted to Phys. Rev. Lett.

[8] J. Igarashi, J. Phys. Soc. Japan 54260 (1985).

[9] F. Aryasetiawan , Phys. Rev. B 46, 13051 (1992)

[10] G. Treglia, F. Ducastelle and D. Spanjaard, J. Phys. Paris 41 281(1980); 43341 (1982).

[11] L. Kleinman and K. Mednick, Phys. Rev. B 246880 (1982).

[12] R.G. Jordan, M.A. Hoyland, Solid State Commun. 72, 433 (1989)

[13] M.M. Steiner, R.C. Albers and L.J. Sham , Phys. Rev. B 4513272 (1992)

[14] A. Liebsch, Phys. Rev. Lett. 431431 (1979)

[15] C. Calandra and F. Manghi , Phys. Rev B 455819 (1992)

[16] Jun-ichi Igarashi, P. Unger, K. Hirai, P. Fulde, Phys. Rev. B 49, 16181 (1994)

[17] C. S. Fadley, D. Shirley , Phys. Rev. A 2, 1109 (1970)

[18] C. Carbone, T, Kachel, R. Rochow, W. Gudat, Solid State Commun. 77, 619 (1991)

[19] T. Kachel C. Carbone, W. Gudat, Phys. Rev. B 47, 15391 (1993) ; C. Carbone, T. Kachel, R. Rochow and W. Gudat, Z. Phys. B 79, 325 (1990)

[20] B. T. Thole, G. van der Laan , Phys. Rev. B 44, 12424 (1991) 
[21] A. K. See and L.E. Klebanoff, Phys. Rev. Lett. 74, 1454 (1995)

[22] Y.Liu, Z. Xu, Pd. Johnson, G. van der Laan , Phys. Rev. B 52, R8593 (1995)

[23] Y. Saitoh, T. Matsushita, S. Imada, H. Daimon, S. Suga, J. Fujii, K. Shimada, Phys. Rev. B 52, R11549 (1995)

[24] L.D. Faddeev, Sov. Phys.- JETP 12275 (1963)

[25] P. H. Dederichs, S. Bluegel, R. Zeller and H. Akai , Phys. Rev. Lett. 53, 2512 (1984)

[26] O. K. Andersen, Phys. Rev. B 12, 3060 (1975)

[27] O. K. Andersen, O. Jepsen , Phys. Rev. Lett. 532571 (1984)

[28] T. J. Kreutz. P. Aebi, J. Osterwalder, Solid State Commun. 96, 339 (1995)

[29] Y. Sakisaka, T. Komeda, M. Onchi, H. Kato, S. Masuda and K. Yagi, Phys. Rev. B 366383 (1987).

FIG. 1. Schematic representation of the basis set for the configuration expansion of the interacting state with one electron removed from the majority-spin band. $\hat{V}, \hat{V}_{h-h}, \hat{V}_{h-e}$ describe the coupling between 1- and 3-particle states, the hole-hole and the hole- electron interaction respectively.

FIG. 2. Schematic representation of the basis set for the configuration expansion of core states.

FIG. 3. Single particle band structure of nickel obtained using the LMTO method. Energies are referred to the Fermi energy. Open triangles, minority-spin states; filled triangles, majority-spin states.

FIG. 4. Density of quasi-particle states of nickel for majority-spin (a) and minority-spin (b) spin compared with experimental results of angle-integrated spin-resolved photoemission results (filled triangles) of ref. [3]. The results for minority-spin bands obtained without the strong-ferromagnetic-limit approximation is shown as a broken line in panel (b).

FIG. 5. Spectral function (a) and self-energy (b) for the $K$ point and $\epsilon_{\mathbf{k} n \uparrow}^{M F}=-3.6$ eV. The interception between $\operatorname{Re}\left(\Sigma^{-}(\mathbf{k} n \uparrow, \omega)\right)$ and the straight line $\omega-\epsilon_{\mathbf{k} n \uparrow}^{M F}$ indicate the position of the quasi-particle pole.

FIG. 6. Quasi-particle band structure of nickel along the high symmetry directions of the Brillouin Zone. Energies are referred to the Fermi energy. Open triangles, minority-spin states; filled triangles, majority-spin states.

FIG. 7. Comparison between the calculated dispersion of hole quasi-particle states (circles) for majority-spin bands and angle-resolved spin-integrated photoemission results (diamonds) of reference [29].

FIG. 8. Self-energy for the creation of majority-spin (a) and minority-spin hole (b) in the core level $3 p^{1 / 2}$.

FIG. 9. Spin-integrated spectral function for the creation of a $3 p$ core hole compared with the photoemission results of ref. [3] (a) and its decomposition into contributions from the spin-orbit split level $3 p^{1 / 2}$ (continuous line) and $3 p^{3 / 2}$ (dashed line). Panel (b) refers to majority-spin state and panel (c) to the minority-spin one.

FIG. 10. (a) Calculated spectrum for the creation of a majority-spin (continuous line) and minority-spin (dashed line) $3 p$ core hole. (b) Difference spectrum between the two spin component.

TABLE I. Parameters used in the calculations of Ni $3 p$ core hole spectrum

\begin{tabular}{cccc}
\hline \hline$U_{c v}$ & $J_{c v}$ & $\epsilon_{c}^{1 / 2}$ & $\epsilon_{c}^{3 / 2}$ \\
\hline $5.00 \mathrm{eV}$ & $2.50 \mathrm{eV}$ & $-59.00 \mathrm{eV}$ & $-57.50 \mathrm{eV}$ \\
\hline \hline
\end{tabular}




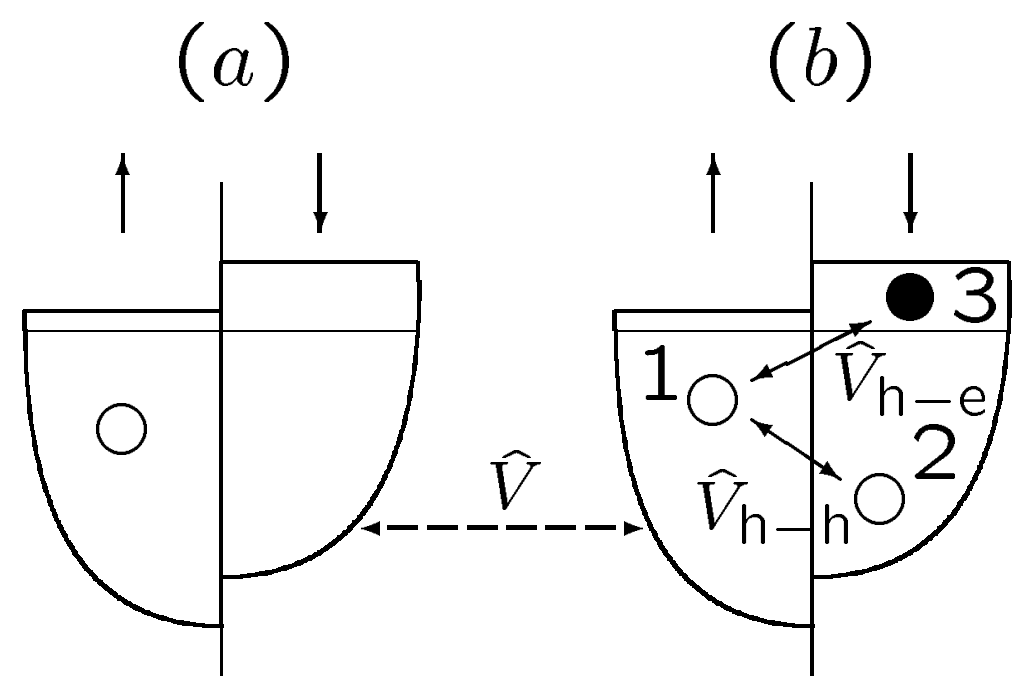




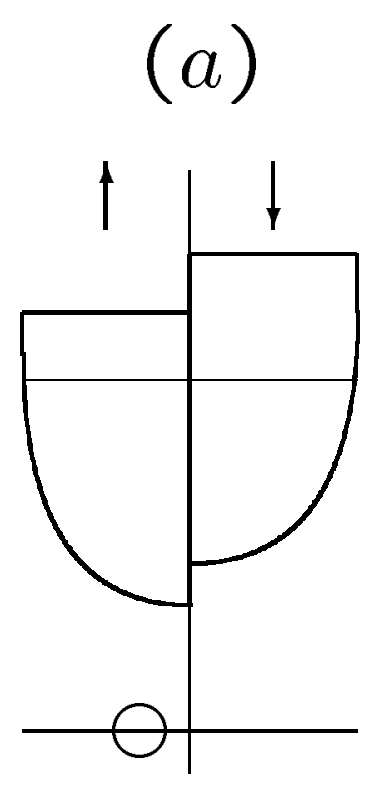

(b)

(c)
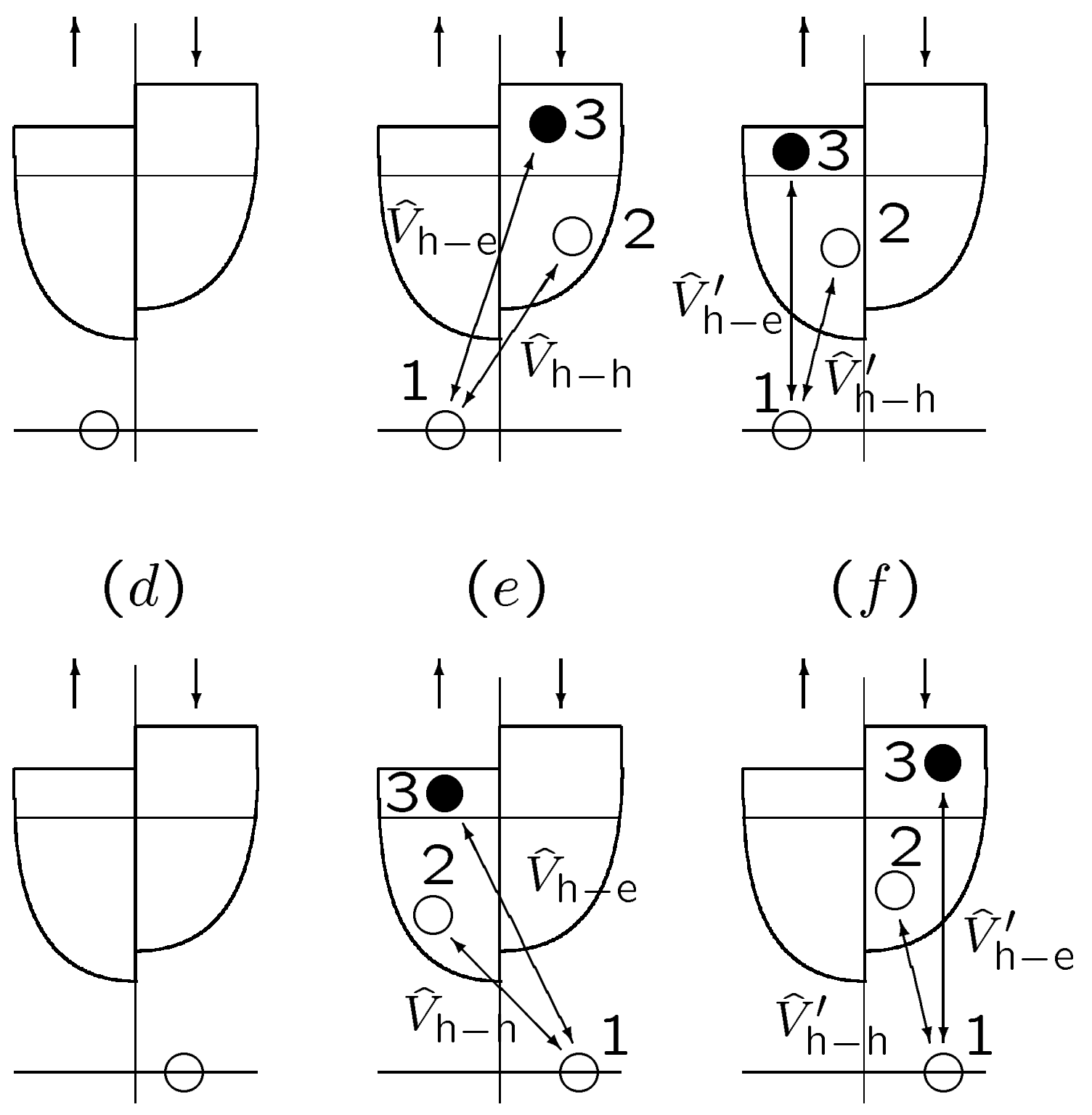


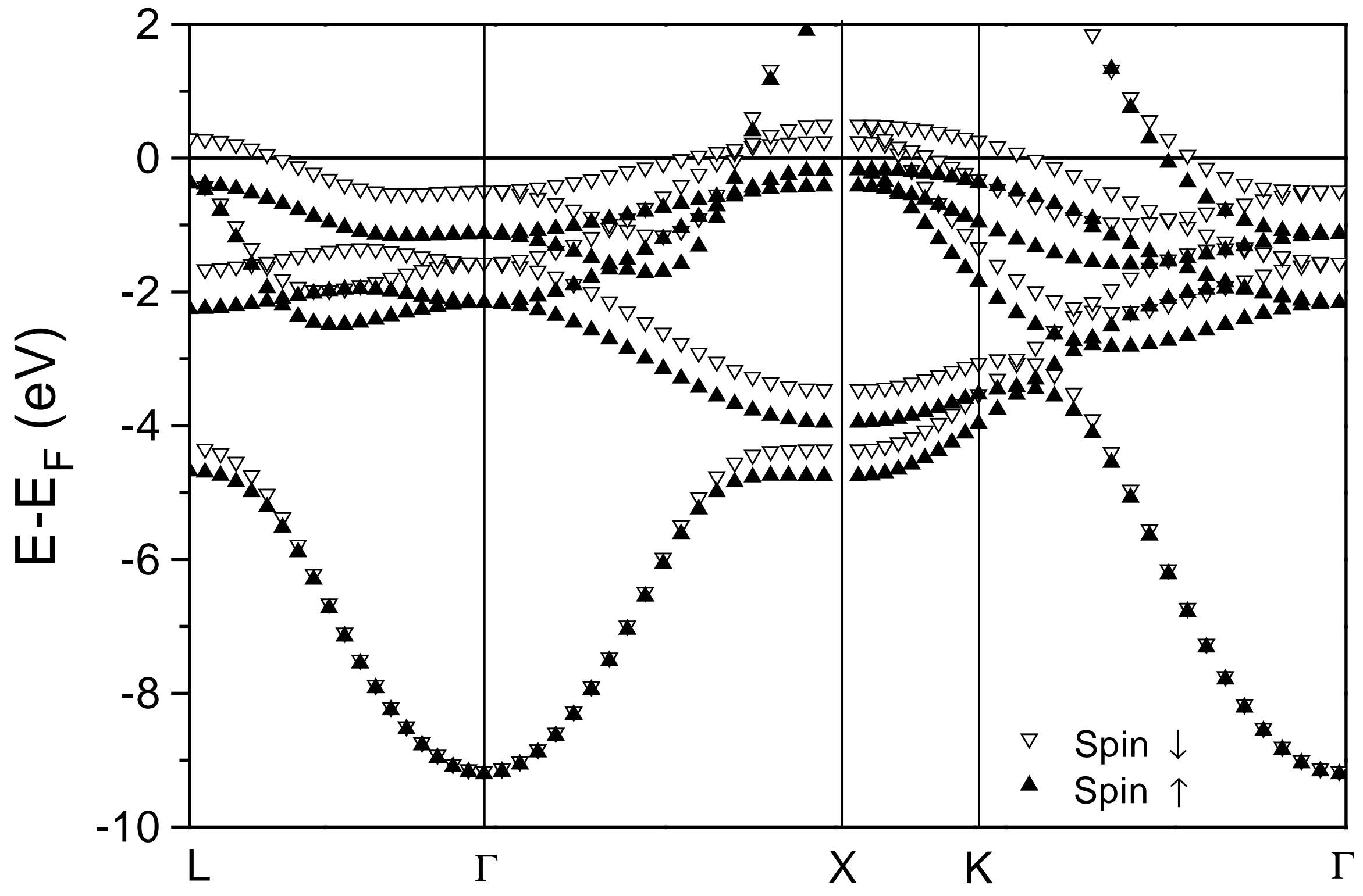




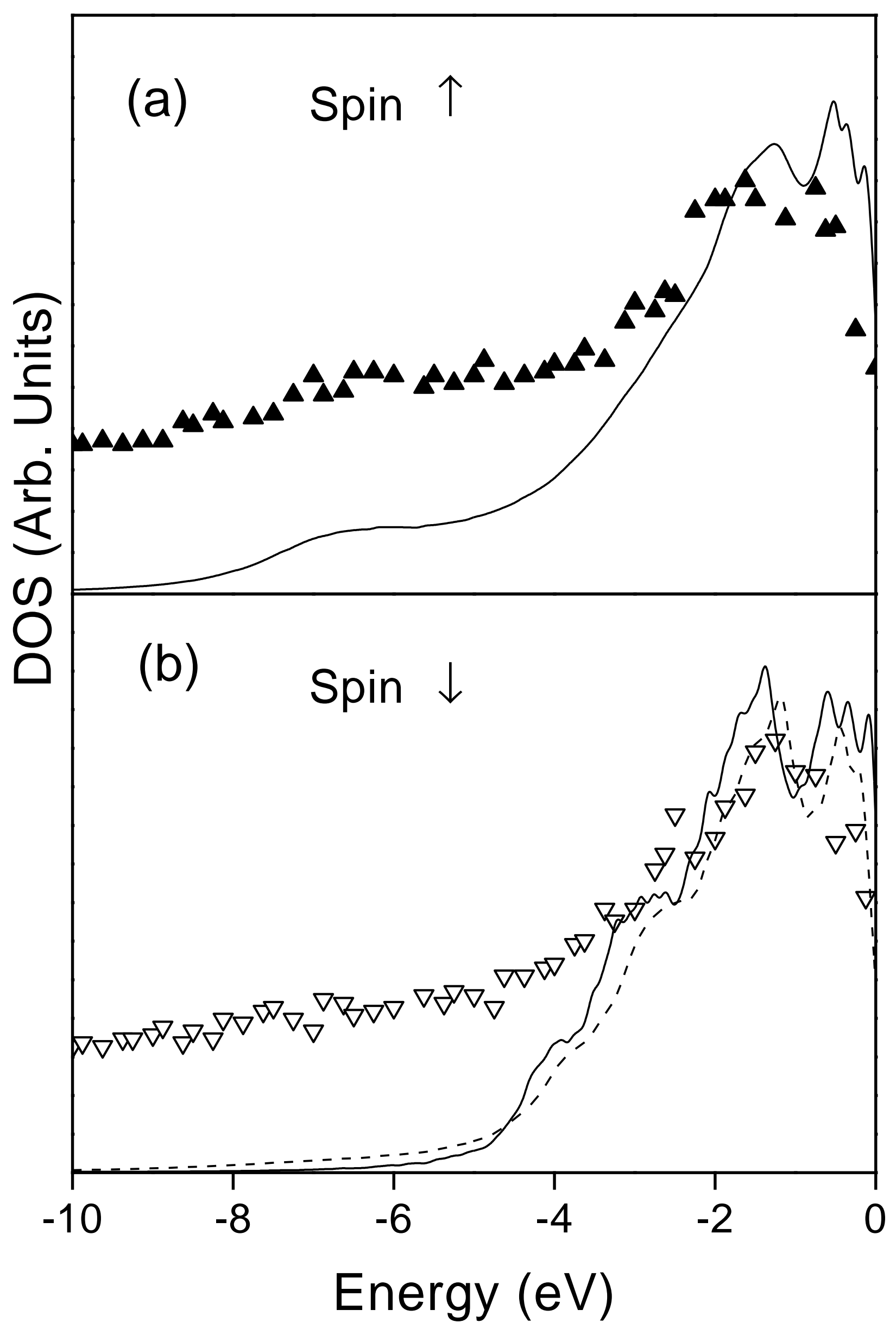




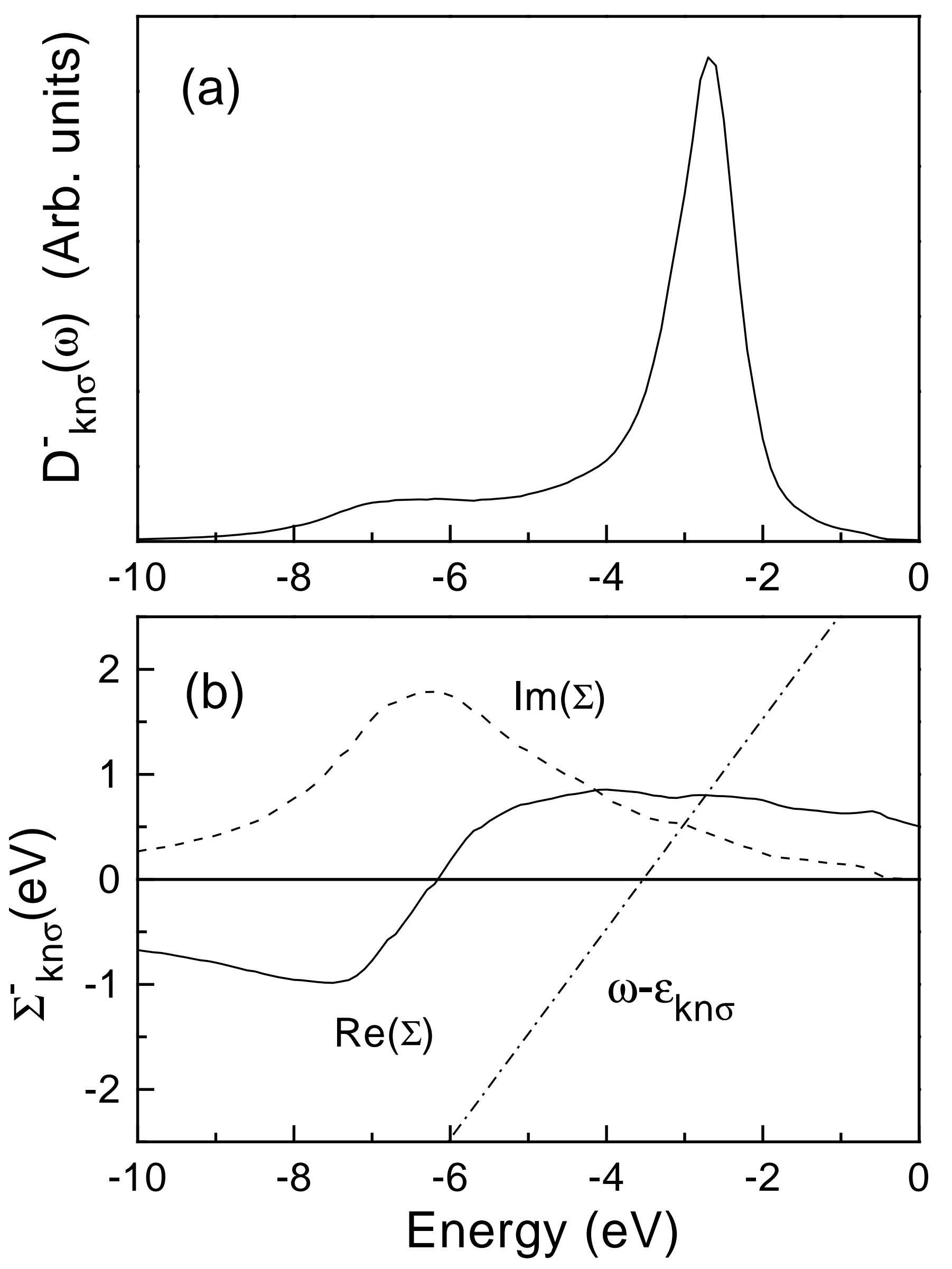




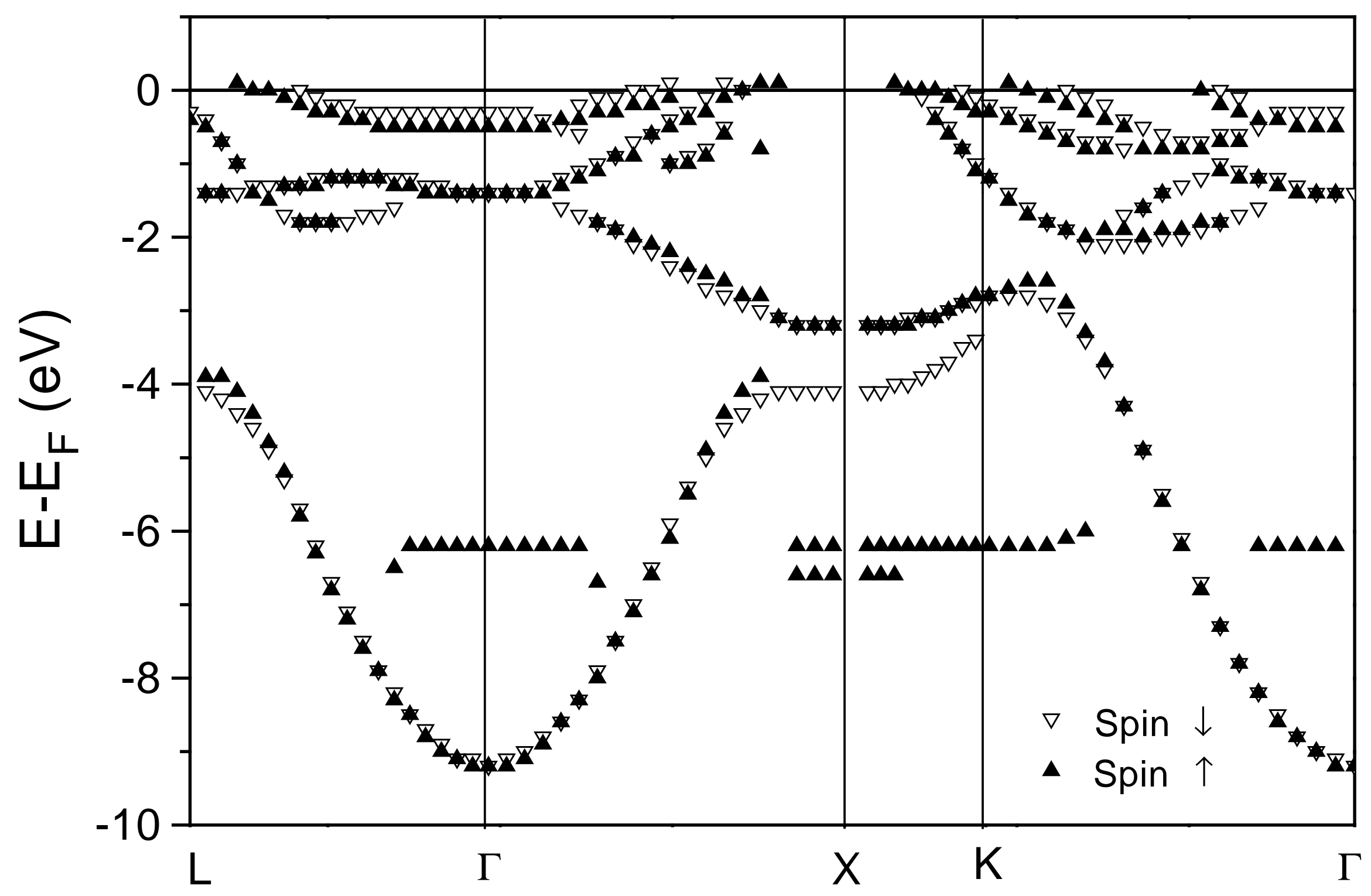




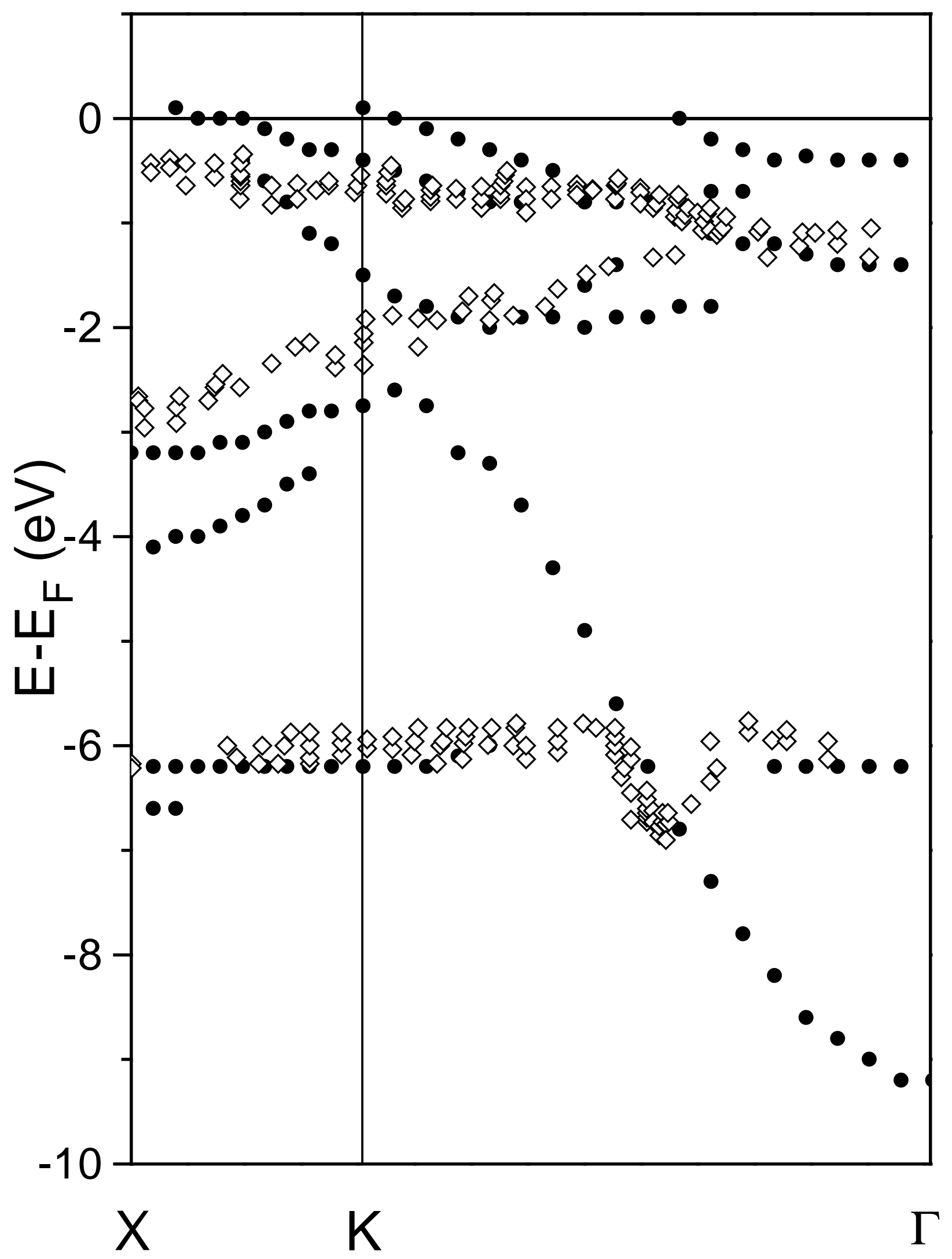




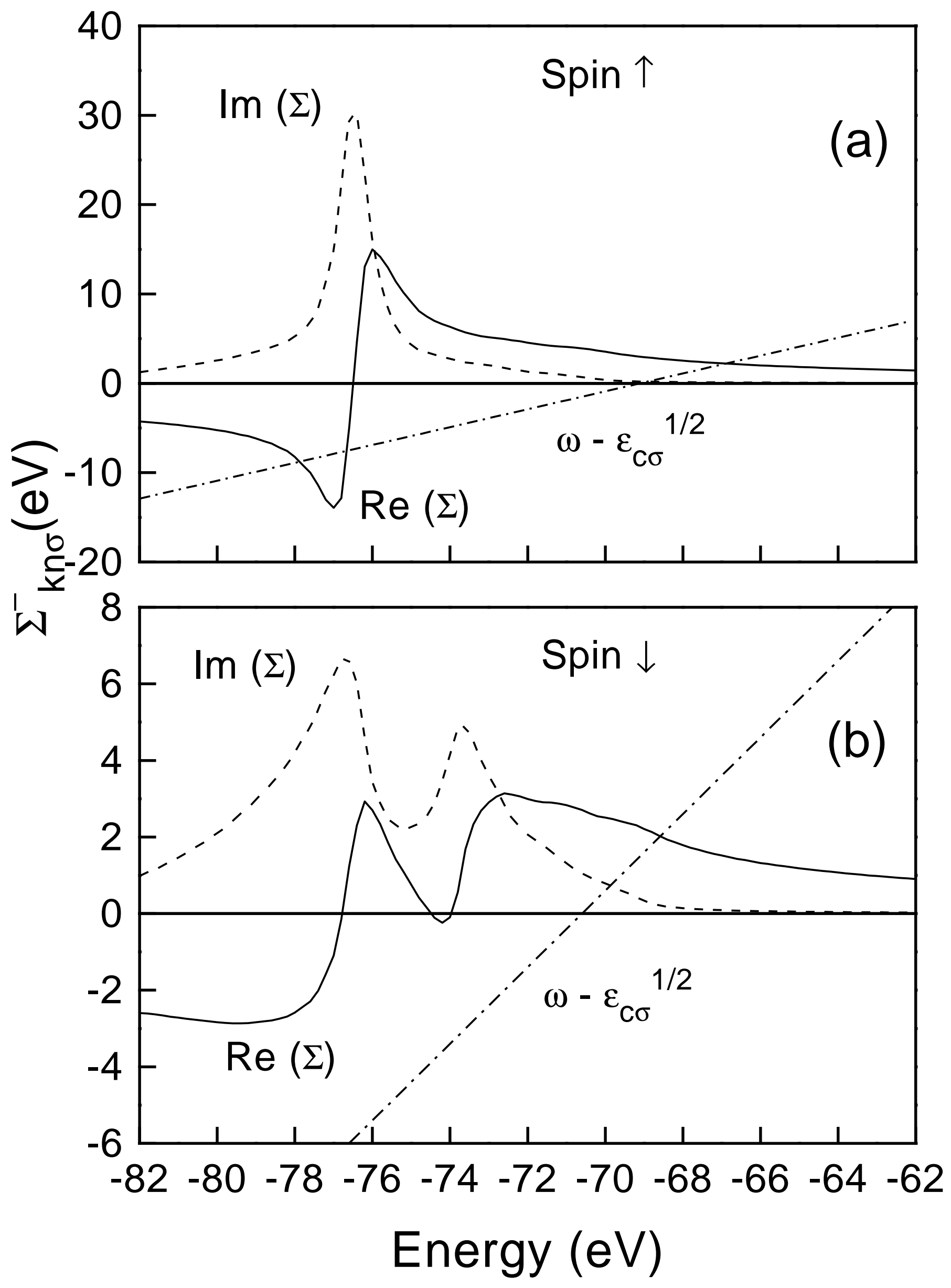




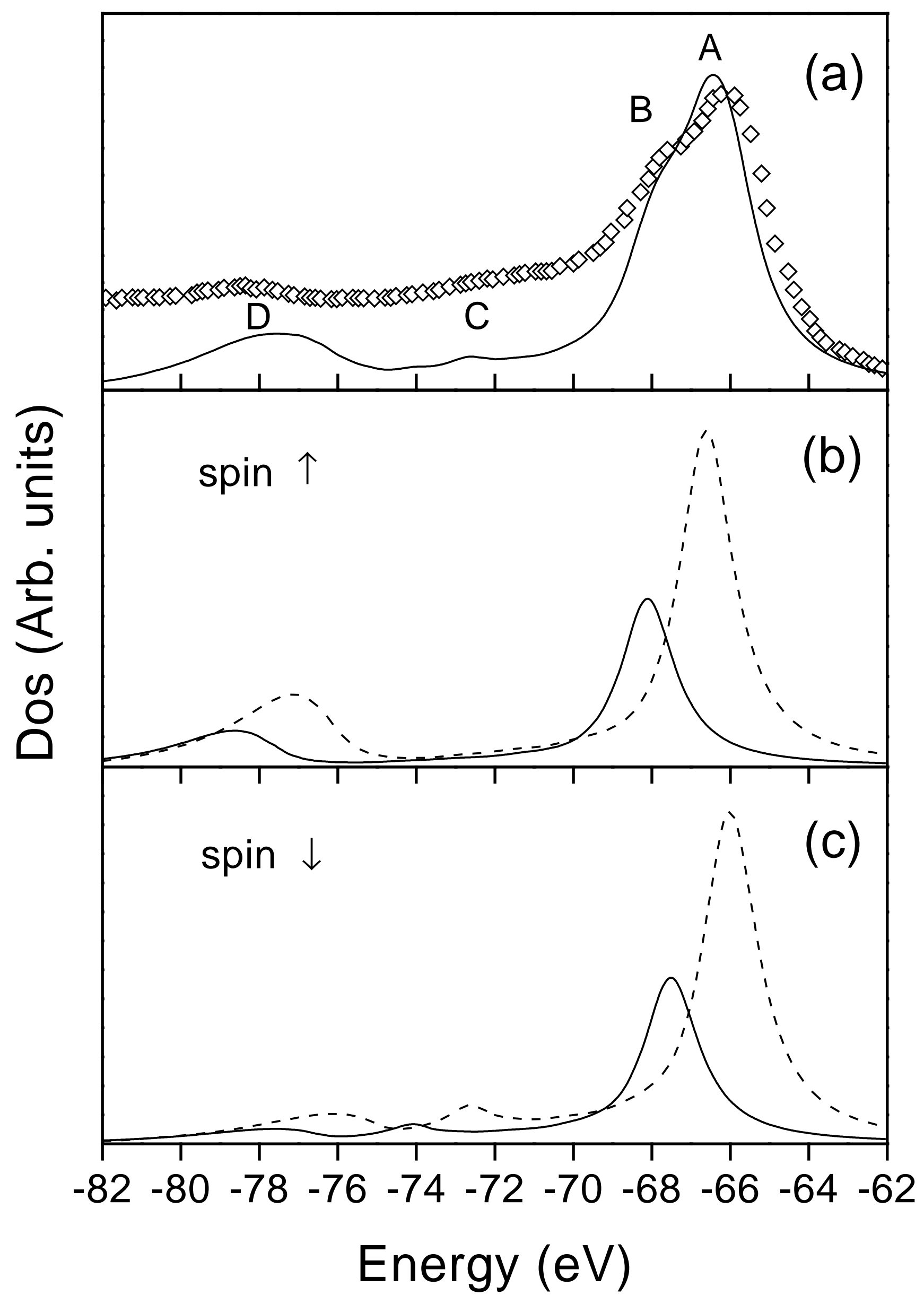



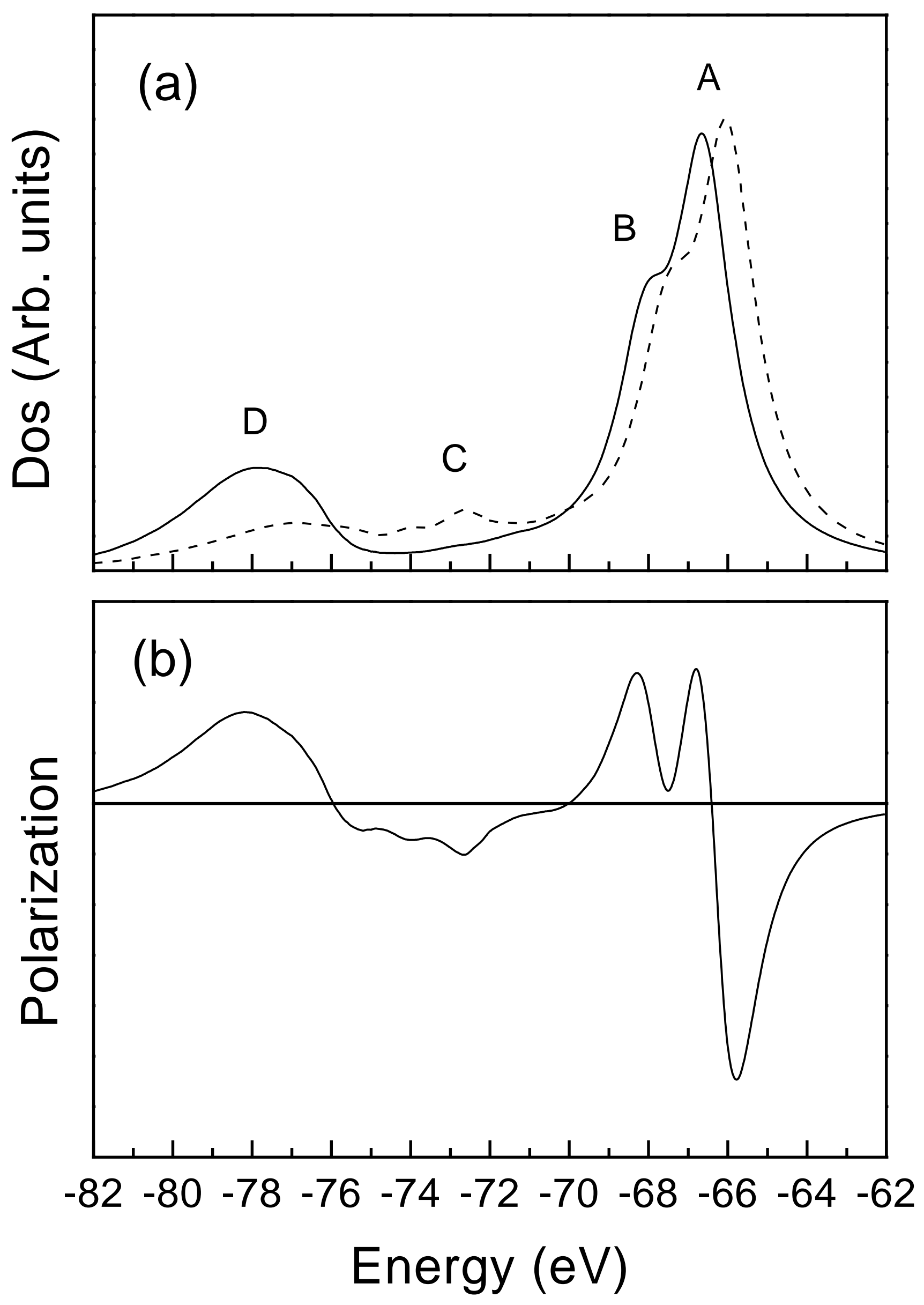